This is the author's final, peer-reviewed manuscript as accepted for publication. The publisher-formatted version may be available through the publisher's web site or your institution's library.

\title{
Present status of microstructured semiconductor neutron detectors
}

Douglas S. McGregor, Steven L. Bellinger, J. Kenneth Shultis

\section{How to cite this manuscript}

If you make reference to this version of the manuscript, use the following information:

McGregor, D. S., Bellinger, S. L., \& Shultis, J. K. (2013). Present status of

microstructured semiconductor neutron detectors. Retrieved from http://krex.ksu.edu

\section{Published Version Information}

Citation: McGregor, D. S., Bellinger, S. L., \& Shultis, J. K. (2013). Present status of microstructured semiconductor neutron detectors. Journal of Crystal Growth, 379, 99110.

Copyright: @ 2013 Elsevier B.V.

Digital Object Identifier (DOI): doi:10.1016/j.jcrysgro.2012.10.061

Publisher's Link: http://www.sciencedirect.com/science/article/pii/S0022024812009864

This item was retrieved from the K-State Research Exchange (K-REx), the institutional repository of Kansas State University. K-REx is available at http://krex.ksu.edu 


\title{
Present Status of Microstructured Semiconductor Neutron Detectors
}

\author{
Douglas S. McGregor*, Steven L. Bellinger, J. Kenneth Shultis \\ S.M.A.R.T. Laboratory, Department of Mechanical and Nuclear Engineering, Kansas \\ State University, Manhattan, KS 66506
}

\begin{abstract}
Semiconductor diode detectors coated with neutron reactive materials have been investigated as neutron detectors for many decades, and are fashioned mostly as planar diodes coated with boron-10 $\left({ }^{10} \mathrm{~B}\right)$, lithium-6 fluoride $\left({ }^{6} \mathrm{LiF}\right)$ or gadolinium $(\mathrm{Gd})$. Although effective, these detectors are limited in efficiency (the case for boron and LiF coatings) or in the ability to distinguish background radiations from neutron-induced interactions (the case for Gd coatings). Over the past decade, a renewed effort has been made to improve diode designs to achieve up to a tenfold increase in neutron detection efficiency over the simple planar diode designs. These new semiconductor neutron detectors are fashioned with a matrix of microstructured patterns etched deeply into the substrate and, subsequently, backfilled with neutron reactive materials. Intrinsic thermal-neutron detection efficiencies exceeding $40 \%$ have been achieved with devices no thicker than $1 \mathrm{~mm}$ while operating on less than 5 volts.
\end{abstract}

Key words:

Semiconductor neutron detectors, solid state neutron detectors PACS: 29.40.Wk

\section{Introduction}

Semiconductor neutron detectors based on thin-film coatings of neutron reactive material (or "converter foils") arranged in close proximity to a semi-

\footnotetext{
${ }^{*}$ Corresponding author.

Email address: mcgregor@ksu.edu (Douglas S. McGregor)

URL: www.mne.ksu.edu/research/centers/SMARTlab (Douglas S. McGregor)
} 
conductor diode have been investigated for many decades [1]-[23]. The first reported devices consisted of simple semiconductor $\mathrm{Ge}$ and $\mathrm{Si}$ diodes coated with ${ }^{10} \mathrm{~B}[1]$. Neutrons interacting in the reactive layer cause the spontaneous ejection of energetic reaction products. Should one of these reaction products enter the adjacent semiconductor diode, it creates numerous electronhole pairs whose charge can be measured through a tiny current or through a shaped voltage pulse. The three most often investigated neutron reactive materials for such a device are ${ }^{10} \mathrm{~B},{ }^{6} \mathrm{LiF}$, or natural $\mathrm{Gd}$.

The thermal-neutron $\left(2200 \mathrm{~m} \mathrm{~s}^{-1}\right)$ capture cross section for ${ }^{157} \mathrm{Gd}$ is 240,000 barns and, consequently, this isotope strongly absorbs thermal neutrons in only a thin film of material. Natural Gd, which has a ${ }^{157} \mathrm{Gd}$ natural abundance of $15.7 \%$, has a thermal-neutron capture cross section of 46,000 barns. However, only $60 \%$ of thermal-neutron captures result in the release of a conversion electron, thereby reducing the effective thermal-neutron cross section of natural Gd to 27,600 barns. The (n, $\gamma$ ) reactions yield numerous low energy conversion electrons, with a general distribution ranging from 29 $\mathrm{keV}$ up to $246 \mathrm{keV} \mathrm{[12].} \mathrm{However,} \mathrm{the} \mathrm{largest} \mathrm{yields} \mathrm{are} \mathrm{from} \mathrm{conversion}$ electrons with energies near $70 \mathrm{keV}$, with all other energy emissions above $85 \mathrm{keV}$ having much lower branching ratios [12]. As a result, the reaction products from Gd are mostly low energy conversion electrons and can easily be confused with background gamma-ray or beta particle interactions. For this reason, Gd coatings are generally not used as a converter for coated semiconductor neutron detectors.

The ${ }^{10} \mathrm{~B}(\mathrm{n}, \alpha){ }^{7} \mathrm{Li}$ neutron reaction yields two possible de-excitation branches from the excited ${ }^{11} \mathrm{~B}$ compound nucleus, namely

$$
{ }_{0}^{1} \mathrm{n}+{ }_{5}^{10} \mathrm{~B} \longrightarrow\left\{\begin{array}{ll}
{ }_{2}^{4} \mathrm{He}(1.4721 \mathrm{MeV})+{ }_{3}^{7} \mathrm{Li}^{*}(0.8398 \mathrm{MeV}) & (93.7 \%) \\
{ }_{2}^{4} \mathrm{He}(1.7762 \mathrm{MeV})+{ }_{3}^{7} \mathrm{Li}(1.0133 \mathrm{MeV}) & (6.3 \%)
\end{array}\right. \text {. }
$$

The $\mathrm{Li}$ ion produced in the $94 \%$ branch is ejected in an excited state, which deexcites through the emission of a $480-\mathrm{keV}$ gamma ray. For thermal-neutron absorptions, the two charged-particle reaction products are ejected in opposite directions. Fully enriched ${ }^{10} \mathrm{~B}$ has a microscopic absorption cross section for thermal neutrons of 3840 barns. With a mass density of $2.15 \mathrm{~g} \mathrm{~cm}^{-3}$, the solid structure of ${ }^{10} \mathrm{~B}$ has a macroscopic thermal-neutron absorption cross section of $500 \mathrm{~cm}^{-1}$. The absorption cross section for ${ }^{10} \mathrm{~B}$ follows a $1 / v$ dependence $[24,25] .{ }^{10} \mathrm{~B}$ has been used successfully as a neutron conversion mechanism on semiconductor diodes for over 50 years, and was used in the 
first demonstration of microstructured semiconductor neutron detectors [26]. The ${ }^{6} \operatorname{Li}(\mathrm{n}, \mathrm{t})^{4} \mathrm{He}$ neutron reaction yields a single product branch,

$$
{ }_{0}^{1} \mathrm{n}+{ }_{3}^{6} \mathrm{Li} \longrightarrow{ }_{1}^{3} \mathrm{H}(2.7276 \mathrm{MeV})+{ }_{2}^{4} \mathrm{He}(2.0553 \mathrm{MeV}) .
$$

For thermal neutrons, the charged particle reaction products are ejected in opposite directions. The reaction products from the ${ }^{6} \mathrm{Li}(\mathrm{n}, \mathrm{t})^{4} \mathrm{He}$ reaction are more energetic than those of the ${ }^{10} \mathrm{~B}(\mathrm{n}, \alpha){ }^{7} \mathrm{Li}$ or ${ }^{157} \mathrm{Gd}(\mathrm{n}, \gamma){ }^{158} \mathrm{Gd}$ reactions and, hence, are much easier to detect and discriminate from background radiations. ${ }^{6} \mathrm{Li}$ has a relatively large microscopic thermal-neutron absorption cross section of $940 \mathrm{~b}$, although less than that of ${ }^{157} \mathrm{Gd}$ or ${ }^{10} \mathrm{~B}$. The absorption cross section for ${ }^{6} \mathrm{Li}$ also follows a $1 / v$ dependence $[24,25]$. Although devices have been fabricated with enriched ${ }^{6} \mathrm{Li}$ metal as the converter film [23], pure $\mathrm{Li}$ is highly reactive and difficult to prevent from decomposing, even with the use of encapsulates. Consequently, the stable compound LiF is more frequently used. The mass density of ${ }^{6} \mathrm{LiF}$ is $2.54 \mathrm{~g} \mathrm{~cm}^{-3}$, and the resulting macroscopic thermal-neutron absorption cross section is $57.5 \mathrm{~cm}^{-1}$.

The advantages of coated diodes as neutron detectors include compact size, low power requirements, low cost VLSI mass production methodology, and ruggedness. Yet, because the basic planar thin-film diode coated with either ${ }^{10} \mathrm{~B}$ or ${ }^{6} \mathrm{LiF}$ can achieve a practical maximum intrinsic thermal-neutron detection efficiency $\epsilon_{t n}$ of approximately only $4.5 \%$ [23], they have not been widely used as neutron radiation detectors. Detectors coated with pure ${ }^{6} \mathrm{Li}$ metal can achieve $\epsilon_{t n}$ greater than about $11 \%$ [23], yet the chemically reactive nature of Li metal complicates their fabrication. Detectors coated with natural Gd foil can achieve $\epsilon_{t n}$ above $22 \%$, but only with the lower level discriminator (LLD) set at zero. With the LLD adjusted up to $80 \mathrm{keV}$, the efficiency of Gd-coated detectors drops below 4\% [12].

It was suggested by Muminov that the incorporation of channels in a semiconductor substrate subsequently backfilled with a neutron reactive material might increase the thermal-neutron detection efficiency of a semiconductor based diode [27]. This idea was also made much later by Schelten $[28,29]$, yet neither group built or reported working devices. Muminov [27] suggested that the extended surface area of the shallow channels would increase the overall detection efficiency, a suggestion that is only partially true. As pointed out elsewhere [30,31,32], it is actually the added effects of the extended surface area and the increased probability that reaction products can enter the semiconductor material, due to additional geometric effects, that truly increases the efficiency. 
The first demonstration of a microstructured semiconductor neutron detector was reported by McGregor et alii [26, 33]. The devices were constructed from bulk GaAs with circular hole perforations backfilled with ${ }^{10} \mathrm{~B}$ [33]. Since then, microstructured semiconductor neutron detectors (MSNDs), also referred to as perforated neutron detectors, have been made with deeper channels and various different perforation geometries [30]-[63].

\section{Modeling Results}

There are fundamentally three variants on the MSND design, although many permutations of these basic patterns have also been explored. The basic designs can be categorized as a matrix of holes filled with neutron reactive material, semiconductor fins between which trenches are filled with neutron reactive material, or semiconductor columns surrounded by neutron reactive material [32], as illustrated in Fig. (1).

Device modeling has been used to determine the best pattern for neutron detection efficiency and for efficiency stability as a function of the LLD; the reader is referred to reference [32] for details. The modeling included a Monte Carlo approach to determine the expected spectral features as a function of the microstructure type, depth, width and unit cell dimension (see Fig. (1) for illustrations of unit cells).

Shown in Figs. (2) to (4) are the expected spectral features in the ion energy-deposition spectrum in Si devices with 40-micron deep trenches backfilled with ${ }^{10} \mathrm{~B}$, in which the fins and trenches are both $2 \mu \mathrm{m}, 4 \mu \mathrm{m}$, or 6 $\mu \mathrm{m}$ wide, i.e., each has a $50 \%$ cell fraction. The $40-\mu \mathrm{m}$ microstructure depth represents two mean-free-path lengths for $2200 \mathrm{~m} \mathrm{~s}^{-1}$ neutrons in ${ }^{10} \mathrm{~B}$. The cell fraction refers to the ratio of the feature dimension to the cell width. For instance, a $2-\mu \mathrm{m}$ diameter hole within a unit cell width (and length) of $4-\mu \mathrm{m}$ has a $50 \%$ cell fraction.

Shown in Fig. (2) are normalized energy-deposition spectra for trench pattern devices. For small dimensions, a wide valley appears in the low energy region (from 0 to approximately $0.6 \mathrm{MeV}$ ) and a prevalent peak is observed in the central portion of the spectrum. For larger unit cells and microstructures, the valley region disappears, a consequence of reaction products being absorbed within the ${ }^{10} \mathrm{~B}$ material ("self-absorption"). A detailed explanation of the features is published elsewhere [32]. Note that background radiations and noise are much easier to discriminate against in devices with wide lowenergy valleys in their spectral features because a higher LLD level can be 
used without appreciable loss of counts. Normalized spectra for hole pattern devices and column pattern devices are shown in Fig. (3) and Fig. (4), respectively. The low energy valley is most prevalent for hole pattern devices (for these geometries), and least prevalent for column pattern devices.

Shown in Figs. (5) to (7) are the expected spectral features for Si devices with 350-micron deep trenches backfilled with ${ }^{6} \mathrm{LiF}$, with feature sizes of $12.5 \mu \mathrm{m}, 25 \mu \mathrm{m}$, and $37.5 \mu \mathrm{m}$, each with a $50 \%$ cell fraction. The $350-\mu \mathrm{m}$ microstructure depth represents two mean-free-paths lengths for $2200 \mathrm{~m} \mathrm{~s}^{-1}$ neutrons in ${ }^{6} \mathrm{LiF}$.

Shown in Fig. (5) are normalized spectra for trench pattern devices. Note that a wide valley again appears in the low energy region (from 0 to approximately $0.7 \mathrm{MeV}$ ) and a peak appears in the central portion of the spectrum. As the cell dimension is increased, the valley separation is still apparent for all cases, unlike the ${ }^{10} \mathrm{~B}$ backfilled devices, a fortuitous consequence of the triton range being over six times greater than the $\alpha$-particle range. However, the efficiency does still decrease as the cell dimensions increase. Normalized spectra for hole pattern devices and column pattern devices are shown in Fig. (6) and Fig. (7), respectively. The low energy valley is most prevalent for hole pattern devices (for the geometries consider here), and least prevalent for column pattern devices, the same as is observed with ${ }^{10} \mathrm{~B}$ backfilled devices.

The fortuitous consequence of a low energy valley region in the spectra is a reduction in the dependence of $\epsilon_{t n}$ on the LLD setting, and an improved gamma-ray rejection ratio (mainly because the LLD can be set relatively high). Shown in Fig. (8) are the modeled efficiency $\epsilon_{t n}$ for ${ }^{10} \mathrm{~B}$ backfilled devices for trenches, holes and columns with $2-\mu \mathrm{m}$ microstructured features in $4-\mu \mathrm{m}$ unit cells, all of which are $40 \mu \mathrm{m}$ deep. With the LLD set at zero, the column pattern device yields the highest efficiency because of the higher amount of absorber present. However, such an LLD setting is unrealistic, and at LLD settings above $80 \mathrm{keV}$, the trench pattern MSND outperforms the other patterns. At an LLD of $950 \mathrm{keV}$, the hole pattern surpasses the trench pattern in performance. Note that the efficiency of the hole pattern is lowest, yet the efficiency is quite stable over a wide range of LLD energy settings, maintaining an $\epsilon_{t n}$ of approximately 16\%-17\% from between LLD settings of zero and $600 \mathrm{keV}$. The trench pattern shows more sensitivity to the LLD setting as the $\epsilon_{t n}$ decreases from $35 \%$ at LLD $=0$ to $27.5 \%$ at 600 $\mathrm{keV}$. The column design is least stable with LLD setting, changing from $37 \%$ $\epsilon_{t n}$ at $\mathrm{LLD}=0$ to $13.5 \% \epsilon_{t n}$ at $600 \mathrm{keV}$. 
Shown in Fig. (9) are the modeled thermal-neutron detection efficiencies for ${ }^{6} \mathrm{LiF}$ backfilled devices for trenches, holes and columns with $12.5-\mu \mathrm{m}$ microstructured features, all of which are $350 \mu \mathrm{m}$ deep. For the trench pattern and hole pattern devices, notice that the change in $\epsilon_{t n}$ is small between LLD settings of 0 up to approximately $0.7 \mathrm{MeV}$, mainly because there are relatively few counts recorded in the pulse height spectra between 0 and 0.7 $\mathrm{MeV}$. For instance, a trench device with $12.5-\mu \mathrm{m}$ wide trench decreases only from $36 \%$ to $35 \% \epsilon_{t n}$ when the LLD is raised from 0 to $0.7 \mathrm{MeV}$, while the hole pattern device maintains an efficiency of approximately $17 \%$ from zero to $900 \mathrm{keV}$. The column pattern device, however, shows a pronounced decrease in $\epsilon_{t n}$ as the LLD is raised from zero, showing a decrease from $39.5 \%$ $\epsilon_{t n}$ at zero to $23 \% \epsilon_{t n}$ at $700 \mathrm{keV}$ equivalent.

There are many cases that can be studied to optimize performance of MSNDs. Examples of spectra and tables of $\epsilon_{t n}$ can be found in the literature [32] for the three basic designs. Overall, ${ }^{6} \mathrm{LiF}$ as a backfill material outperforms ${ }^{10} \mathrm{~B}$ for several reasons. First, the higher energy reaction products from the ${ }^{6} \mathrm{Li}(\mathrm{n}, \mathrm{t})^{4} \mathrm{He}$ reaction causes more energy to be deposited in the adjacent semiconductor walls. Second, the longer ranges of ${ }^{6} \mathrm{Li}(\mathrm{n}, \mathrm{t})^{4} \mathrm{He}$ allow for larger MSND dimensions, thereby, making device fabrication much easier. Third, the LLD can be set higher for ${ }^{6} \mathrm{Li}$-backfilled detectors than for ${ }^{10} \mathrm{~B}$-backfilled detectors without losing significant neutron interaction events, mainly because the low energy valley is much wider, again due to the higher energies of the reaction products.

Other designs that utilize hole patterns or column patterns are less interesting, either because of limited efficiency or poor efficiency stability [32]. Microstructured designs using small circular holes show good stability in performance as a function of the LLD setting, thereby, offering larger signal to noise ratios; however, these patterns generally deliver lower efficiencies. If the hole diameters and cell fractions are increased, the efficiency improves, but the discrimination stability becomes much worse, similar to the performance of detectors with column patterns. Microstructured designs with columns generally show improved efficiency for low LLD settings, provided that the structural features are very small. Unfortunately, column pattern designs show large changes in efficiency as a function of the LLD setting. By contrast, the trench and circular hole designs have a much more stable $\epsilon_{t n}$ as a function of LLD [32].

Finally, it should be noted that these modeling calculations are "ideal" in the sense that they do not include processing effects. Damage to side- 
walls, oxidation and dopant diffusion causes a dead layer to appear along the surfaces in the microstructures. This dead layer absorbs reaction product energy and cause the pulse height spectra to shift into lower energy channels. This effect is more pronounced as the features sizes decrease, mainly because a larger percentage of the semiconductor material is consumed by the perforations. MSND devices fabricated with ${ }^{10} \mathrm{~B}$ backfilling must have small features on the order of $1-3 \mu \mathrm{m}$ to yield adequate efficiency, and this causes pulses to accumulate in low energy channels and compromises both $\epsilon_{t n}$ and the gamma-ray discrimination capability [49, 64]. MSND devices fabricated with ${ }^{6} \mathrm{LiF}$ backfilling can have features on the order of $10-30 \mu \mathrm{m}$ and yield good efficiency. As a result, processing damage has less effect on the spectra $[62,63]$.

\section{Detector Development}

The processes used to fabricate MSNDs have matured much over the past decade such that the methods are now inexpensive and reliable. Each fabrication process involves, at some level, the etching of a semiconductor substrate, the fabricating of a rectifying junction upon the substrate, and the backfilling of the microcavities with neutron reactive material. A brief overview of the history of development and present status is reviewed in the following sections.

\subsection{Etching}

Substrate etching generally involves the application of a pattern, typically with photoresist, and the subsequent removal of substrate material within the exposed regions of the pattern. Two basic methods have been explored, namely, dry etching and wet etching. The main considerations for etching methods include selectivity, anisotropy (aspect ratio), etch rate, and surface damage. The selectivity refers to the ratio at which the substrate material is removed as opposed to the protective masking material. Anisotropy is the ratio of the perforation depth to the perforation width. For instance, the aspect ratio for a $20 \mu \mathrm{m}$ wide trench that is $460 \mu \mathrm{m}$ deep is 1:23. Deep microstructured features require high anisotropy etch methods. Etch rate refers to the rate at which material is removed from the substrate. For eventual commercialization purposes, a high etch rate is preferred. Finally,

the damage to the microstructured feature surfaces must be minimal in order to suppress leakage currents and minimize the thickness of dead layers. 


\subsubsection{Dry Etching}

Dry etching uses an energetic plasma of ions to create the perforations in the semiconductor material. The plasma may consist of non-reactive ions, as typically used in a technique referred to as ion milling, or the plasma may consist of chemical etchants, commonly referred to as reactive ion etching. The first technique used to produce MSNDs was capacitive reactive ion etching (REI) of GaAs substrates, in which 4- $\mu \mathrm{m}$ diameter holes were etched up to $5 \mu \mathrm{m}$ deep into semi-insulating GaAs $[26,33]$. The etching chemistry consisted of a plasma composed of $\mathrm{Ar}$ and $\mathrm{BCl}_{3}$ gases [26, 33]. The capacitively coupled RIE process proved to be slow, etching only a few microns per hour, and was later replaced by the inductively coupled plasma (ICP) reactive ion etching (REI) method.

ICP-RIE is a far more aggressive etching approach, producing etch rates exceeding a hundred microns per hour. Further, the selectivity is considerably higher than that achievable with capacitively coupled RIE. The ICP-RIE method is now commonly used to produce MSNDs. Typically, the ICP-RIE technique uses a passivation method to protect the sidewalls of the features as the etch progresses. After a short etch, the etching gases are evacuated and a passivation layer is applied. The etch gas is then reintroduced, followed by a short etch time before the next passivation cycle, a cycling process that continues until the desired depth is reached. Unfortunately, as the features increase in depth, the cycling time must also change in order to allow for efficient evacuation of the etch gas and deposition of the passivation layer. For the depths required for MSNDs, dry etching problems such as "tapering", "bottling", "scalloping", "ribbing" and "vertical striations" that degrade device performance $[60,64,65]$. These effects, many of which are shown in Figs. (10) to (13), are caused by fluctuations in the chemical plasma concentration during processing, and the nature of the alternating etch-deposit method. Overall, the deep features with high aspect ratios needed for MSNDs are difficult to achieve reliably and repeatedly. Further, it is especially difficult to maintain smooth sidewall surfaces that are necessary to reduce surface recombination currents.

Regardless, etched features deeper than $300 \mu \mathrm{m}$ with aspect ratios $>1: 10$ have been achieved with ICP-RIE [60]. For Si etching, ICP-RIE chemistry typically includes a combination of $\mathrm{SF}_{6}$ as the reactive gas followed by the deposition of $\mathrm{C}_{4} \mathrm{~F}_{8}$ as the passivation layer. For GaAs etching, ICP-RIE chemistry typically includes a combination of $\mathrm{Cl}_{2}$ and $\mathrm{BCl}_{3}$ as the etching gases. 
Unfortunately, the ICP-RIE method is ultimately too slow for large batch processing of MSNDs, even with the high etching rates that are achievable, mainly due to throughput issues. Although commercial reactors can batch process many wafers at a time, the systems must be cleaned between long etch runs in order to maintain process stability.

Perhaps the main advantage of ICP-RIE is that almost any pattern can be etched into a semiconductor substrate, provided that the feature dimensions are not too small. Various patterns have been developed, based on the trench design, including holes, sinusoids, and chevrons [52]. Column pattern devices have been fabricated with limited success, mainly due to excessive leakage current and surface dead layers most likely caused by etching damage [49]. Hole pattern devices processed with ICP-RIE are also under investigation, but show irregular etched surfaces and scalloping, effects that appear to limit and compromise device performance [64].

Overall, ICP-RIE works well for shallow microstructures, but the wall thickness and smoothness are difficult to control for the aspect ratios and depths needed to mass produce high efficiency MSNDs. The difficulties apply to both ${ }^{6} \mathrm{Li}$ and ${ }^{10} \mathrm{~B}$ backfilled detectors.

\subsubsection{Wet Etching}

The first published attempt at producing etched features for an MSND reported the use of a potassium hydroxide $(\mathrm{KOH})$ etch $[28]^{1}$. The authors of [28] did not report on the Si wafer properties or etch conditions; however, $20-\mu \mathrm{m}$ wide and $160-\mu \mathrm{m}$ deep trenches were produced to produce an aspect ratio of 1:8. The paper did not report completed or tested MSNDs.

Wet etching results reported by the authors and their colleagues show significant improvement over dry etch methods with aspect ratios greater than 1:23 [59, 61, 62]. The method allows for straightforward batch processing at low cost, with etch rates exceeding $1.5 \mu \mathrm{m}$ per minute in Si. The technique requires the use of (110) oriented Si wafers, in which trench features are aligned along the (111) crystal planes on the wafer surface. Aqueous $\mathrm{KOH}$ has a large etch selectivity, often exceeding 1:300 [65]-[67], when the trench features are properly aligned to the (111) planes on a (110) Si wafer. In the present work, Si wafers are etched in a $50 \% \mathrm{KOH}$ by volume solu-

\footnotetext{
${ }^{1}$ Also, in that particular work, the authors suggested the use of a diamond saw to produce microstructures [28]; however such an approach is not advisable due to the excessive surface damage produced in the microstructures.
} 
tion at $60^{\circ} \mathrm{C}$. The etched features are typically far more uniform than can be achieved with ICP-RIE, and the surface damage is significantly reduced below that observed with ICP-RIE. The drawback to $\mathrm{KOH}$ etching is that it limits the patterns that can be used mainly to rectangular shapes such as straight trenches. Because the (111) planes are oriented at an angle of 109.47

- to each other, etching square holes or columns is difficult. The problem is worsened by dangling bonds at the apex of the angle between converging (111) planes, which work to reduce etch selectivity. Hence, wet etching is best used for the straight trench pattern MSNDs, yet another reason why the trench pattern is superior to the other basic perforation patterns.

\subsection{Diode Fabrication}

There are efforts from other groups to manufacture various microstructured patterns $[46,49,64]$, most of which show increased noise in the spectra. Earlier generation MSNDs fabricated by the authors [36, 52] had similar issues, and were determined to be caused by, at least in part, from surface damage along the microstructure sidewalls. As a result, the LLD must be increased well above the noise level to reduce dead time resulting from electronic noise and leakage currents, a necessity which unfortunately decreases $\epsilon_{t n}[32]$.

Several approaches have been used to reduce leakage current in the basic design, including selectively diffusing $p$-type dopants (in $n$-type material) around the microstructures and growing a thermal oxide inside the microstructures to increase the surface resistance[36, 38, 51], thus, slightly complicating the fabrication process $[36,38,51]$. However, the pulse height spectra from these selective-diffused devices do not match the basic modeled characteristics shown in Fig. (5) (and detailed in [32]); hence it is possible that surface damage caused from the processing steps, inadvertent doping, or perhaps other processing complications prevented full depletion of the device while still causing some spectral contamination from electronic noise [52].

An alternative method for making the microstructured semiconductor neutron detectors requires diffusing the $p n$ junction inside the trench structure $[42,51,53,57,60,62,63]$. The diode contact geometry is no longer produced on the primary pre-etched surface, but conforms to the surface of the trench structure as shown in Fig. (16). This fabrication method consumes etch damage in the perforation, hence, it is not affected by the etched surface and does not require the oxide passivation step. Ultimately, the conformal diffusion of a rectifying junction within the microstructures has provided the 
best reported results [51, 53, 60, 62, 63], and produces the lowest leakage currents (see Fig. (17)) and superior definition in the pulse height spectra $[51,53,60,62,63]$. As pointed out in the literature, the drawback to the conformal diffusion method is that the electric field inside the semiconductor material forming the microstructures is reduced, thereby, reducing charge carrier velocities $[59,62]$. Hence, the integration time on the electronics must be increased in order to collect the ioninduced charges [59, 62]. The conformal diffusion method is now being adopted by other groups working on MSNDs [64].

\subsection{Backfilling}

The authors must report that the most frequent concern from skeptics of the MSND technology are some doubts that an economical method of backfilling the microstructures could be developed. Over the past ten years, various backfilling methods have been explored, including physical vapor deposition, ultrasonic deposition of particles, handpacking, rapid thermal melting, plasma deposition, low pressure condensation, and centrifugal filling.

The original MSNDs were backfilled by physical vapor deposition, where $98 \%$ enriched ${ }^{10} \mathrm{~B}$ was evaporated by electron beam into $3.5-\mu \mathrm{m}$ diameter $5-\mu \mathrm{m}$ deep holes [33]. However, for deep structures, the evaporation method causes the holes to pinch close, as reported in [33] and shown in Fig. (18). Hence, physical vapor deposition works only for low aspect ratios and shallow features. Ultrasonics have been used to backfill microscopic powders of ${ }^{10} \mathrm{~B}$ and ${ }^{6} \mathrm{LiF}$ into microstructures [33]; however, the method also does not work for deep microstructures, or microstructures with high aspect ratios. Closely akin to evaporation are the plasma deposition methods, which have also been tried $[49,61,64]$. A major difference is the plasma deposition provides a more conformal application of the material than evaporation. However, similar to evaporation, the techniques used thus far appear to leave voids inside the microstructures, thereby, reducing $\epsilon_{t n}$. Further, plasma deposition methods are generally slow, which might be adequate for prototyping devices in a laboratory, but is not necessarily the best choice for mass producing detectors. Overall, the methods cited in the literature to backfill ${ }^{10} \mathrm{~B}$ include evaporation, ultrasonic powder, forced powder and plasma deposition, all of which have shown less than ideal results.

Forcing LiF powder into microstructures with pressure has been used, and somewhat successful for features no deeper that $200 \mu \mathrm{m}$ (see Fig. (19)). 
Friction prevents forcing LiF powder into microstructures deeper than approximately $200 \mu \mathrm{m}$ without the risk of damaging the delicate semiconductor features. Further, the packing of the powder, because of variations in grain sizes, is usually not optimal, i.e., it reduces the packing fraction of the neutron absorber in the microstructures. An attempt to remedy this problem included rapid thermal annealing of LiF in the microstructures, as shown in Fig. (20). This approach worked quite well to remove void space and completely fill the structures; however, the temperatures required to perform the operations served to also contaminate the $p n$ junction, and, thereby, destroying the devices. A nanopowder method was also explored, referred to as low-pressure condensation (LPC) $[41,57]$, in which LiF evaporated in a furnace tube is transported to a chilled semiconductor surface where it condenses in the microstructures [41]. This method works well and because it completly fills the microstructures; however, it is also very slow, taking several hours to complete a single wafer of MSNDs.

Most recently developed and the best method to backfill microstructures, to date, is to use a combination of ultrasonics, LPC and centrifugal forcing [69]. LPC is used to produce the ${ }^{6} \mathrm{LiF}$ nanoparticles [60]. Afterwards, the nanoparticles are added to a solution and ultrasonics are used to disperse the nanoparticles and create a colloidal suspension. A wafer of processed MSNDs is then placed in the same container as the suspension, and the container is centrifuged at several thousand rpm. The ${ }^{6} \mathrm{LiF}$ nanoparticles are forced deep into the microstructures, completely filling them, as shown in Fig. (21). The centrifuge operation takes no more than 30 minutes to fill microstructures over $450 \mu \mathrm{m}$ deep, without damaging the delicate detector features or the electronic integrity of the diode structure.

\subsection{Detector Fabrication}

MSNDs are fabricated using a combination of processes that permit the relatively rapid mass production of high performance devices. These processes include wet etching with $\mathrm{KOH}$, conformal dopant diffusion inside the microstructures and centrifugal backfilling of ${ }^{6} \mathrm{LiF}$ in the microstructures. Individual MSNDs are currently batch-processed on 4-inch diameter highresistivity $10 \mathrm{k} \Omega$-cm $n$-type (110) Si wafers from Topsil. Initially, the silicon wafers are chemically cleaned with a Piranha ${ }^{2}$ bath at $130{ }^{\circ} \mathrm{C}$ for 15 minutes

\footnotetext{
${ }^{2} 1 \mathrm{H}_{2} \mathrm{O}: 1 \mathrm{H}_{2} \mathrm{SO}_{4}: 1 \mathrm{H}_{2} \mathrm{O}_{2}$.
} 
to remove organic contaminants, followed by a Baker Clean ${ }^{\circledR 3}$ bath at $70{ }^{\circ} \mathrm{C}$ for 15 minutes to remove metallic and particle contaminants. The residual oxide is then removed from the wafers with a 30-second dip in a buffered oxide etch (BOE) solution. ${ }^{4}$ The wafer is immediately spin-rinsed and placed in a processing furnace. All MSND wafers are cleaned with this process before any high temperature processing is completed, e.g., oxidation and diffusion processing.

A $2.5-\mu \mathrm{m}$ thick wet-oxide is initially grown on a clean wafer. A square diffusion-window is then lithographically patterned with one edge of the square-window aligned to the primary flat and the pattern centered on the wafer. The oxide is then partially removed to a thickness of $2 \mu \mathrm{m}$ via BOE wet-etching. A second straight trench pattern is then lithographically placed on the wafer, aligned within the square diffusion windows, and positioned such that the length of the trenches are parallel to the primary flat, i.e., the trench pattern, masked in the residual oxide within the diffusion window, is aligned to the primary flat direction $\langle 111\rangle$ on the (110) oriented $\mathrm{Si}$ wafer. The rest of the initial oxide in the trench pattern windows is then removed via $\mathrm{BOE}$ wet-etching.

Trenches are then wet-etched into the Si wafer with a $50 \%(\mathrm{w} / \mathrm{v})$ aqueous $\mathrm{KOH}$ wet-etch submersion process at $60{ }^{\circ} \mathrm{C}$ to the desired depth. Individual detectors are currently designed with active areas between 1 and $4 \mathrm{~cm}^{2}$ and have straight trenches etched to a width, pitch, and depth that maintain high neutron-detection efficiency while creating an opportunity to off-set and stack the detector chips to maximize neutron absorption.

After the $\mathrm{KOH}$ wet-etch process, the wafer is chemically cleaned with an $\mathrm{HCl}$ bath $^{5}$ at $70{ }^{\circ} \mathrm{C}$ for 15 minutes to remove residual potassium, followed by a clean with the standard aforementioned chemical clean. The wafer is then BOE wet-etched until the trench masking oxide within the diffusion window is removed. $P$-type regions are then diffused uniformly into the individual device microstructures across the wafer, thereby forming $p n$ junctions within the trenches. After processing, the oxide on the backside of the wafer is removed through a $\mathrm{BOE}$ wet-etch and a $\mathrm{Ti}-\mathrm{Au}$ metal contact is evaporated on the backside of the wafer to make an electrical ground contact, thus,

\footnotetext{
${ }^{3} 25 \mathrm{H}_{2} 0: 5$ BakerClean ${ }^{\circledR}: 1 \mathrm{H}_{2} \mathrm{O}_{2}$.

${ }^{4} \mathrm{JT}$ Baker ${ }^{\circledR}$ buffered oxide etch $6: 1$, an aqueous $\mathrm{NH}_{4}: \mathrm{HF}$ etchant solution.

${ }^{5} 5 \mathrm{H}_{2} \mathrm{O}: 1 \mathrm{HCl}: 1 \mathrm{H}_{2} \mathrm{O}_{2}$.
} 
completing the diode structure and enabling depletion through the bulk of the individual MSNDs. Finally, ${ }^{6} \mathrm{LiF}$ nano-powder is centrifugally backfilled into the microstructures to function as the neutron-absorbing converter material.

\section{Performance of Detectors}

Large-area $4-\mathrm{cm}^{2}$ individual and stacked MSNDs have been mounted in two identical detector systems as described elsewhere [62] (see Fig. (22)). In the configuration for the stacked MSNDs, the anodes of each detector are common and applied to the input of the charge sensitive preamplifier. A relatively short time constant is used because high counting rates are expected for some applications. The preamplifier board can also be reconfigured such that the feedback from a downstream integrator can be applied to compensate for the large leakage currents that may result from the detector processing steps. Because the detectors are photosensitive, the individual MSND and stacked MSNDs, along with the motherboard, must be placed in a shielded lighttight box.

The stacked-detector thermal-neutron counting efficiency was measured with a $0.0253-\mathrm{eV}$ diffracted neutron beam from the Kansas State University TRIGA Mark II nuclear reactor. The neutron flux was calibrated with a Reuter-Stokes ${ }^{3} \mathrm{He}$ gas-filled proportional detector and found to be $1.05 \pm$ $0.02 \times 10^{4} \mathrm{~cm}^{-2} \mathrm{~s}^{-1}$. Details of the calibration method can be found elsewhere $[53,70]$. In addition, by using a diffracted thermal-neutron beam, a direct comparison can be made between the experimental performance of the MSNDs and that of the modeled thermal-neutron detection efficiencies and ion energy-deposition spectra, which are reported elsewhere [32, 58]. A pulse-height spectrum was collected from the individual and stacked MSNDs in the diffracted neutron beam with and without a beam blocking $\mathrm{Cd}$-shutter so as to allow the collection of neutron responses with and without thermalneutrons. Prompt gamma-rays emitted from the thin Cd-shutter appear in the spectrum as numerous pulses at low energy near the noise floor of the detector system (see Fig. (23)). Because the measurement was performed near a nuclear reactor, there is an appreciable neutron background observed as counts above channel 25 in the background measurement. For pulse height spectra collected from either individual MSND or the stacked MSNDs (with an lower level discriminator (LLD) set above the system electronic noise), the neutron counting efficiency was calculated by dividing the summed spectrum neutron count rate by the calibrated neutron flux measured with a ${ }^{3} \mathrm{He}$ 
detector.

So as to allow the collection of responses with and without thermalneutrons, a pulse-height spectrum was collected from an individual $4-\mathrm{cm}^{2}$ MSND (with a 10- $\mu$ s charge-integration time) in the diffracted neutron beam with and without a beam blocking Cd-shutter. Because the diffracted neutron beam is not composed purely of thermal neutrons [71], it is possible that some epi-thermal and fast neutrons, not absorbed in the Cd-shutter, are measured by the MSND. The $4-\mathrm{cm}^{2}$ MSND operated on 1 volt of reverse bias. Note in Fig. (23) that the pulse-height spectrum is significantly separated from the noise/gamma-ray contribution. Although the single MSND is two times larger than previously stacked $1-\mathrm{cm}^{2}$ MSNDs [62], the charge collection is still complete and, thus, forms large distinguishable neutron interaction pulses. In addition, the MSND exhibits the expected spectral shape predicted elsewhere [32], with a pulse-height frequency dip at low energy.

For the $4-\mathrm{cm}^{2}$ large-area MSND design, with the LLD set to channel 25 (slightly above the majority of the background counts), the intrinsic thermalneutron detection efficiency was measured to be $16.33 \pm 0.09 \%$. For the MSND design, with the LLD set to channel 32, the intrinsic thermal-neutron detection efficiency was measured to be $15.94 \pm 0.09 \%$. Note that increasing the LLD from channel 25 to 32 , that $\epsilon_{t n}$ only decreased by $0.39 \%$ in efficiency. This dip in the pulse height spectrum in the low-energy region is vitally important for retaining neutron detection efficiency while effectively discriminating against background radiation.

To further improve neutron detection efficiency, individual large-area 4$\mathrm{cm}^{2}$ MSND chips were stacked back-to-back, as was done with early generation 1-cm ${ }^{2}$ MSNDs [62]. Dual-integrated neutron counting efficiency was measured for a stacked MSND configuration of two $200 \mu \mathrm{m}$ deep, $4-\mathrm{cm}^{2}$ area MSNDs. The stacked dual-integrated MSNDs operated with 1 volt of reverse bias. The combined leakage current for the $4-\mathrm{cm}^{2}$ stacked MSNDs was 78 $\mathrm{nA}$. A pulse-height spectrum from a dual-integrated, stacked, 4-cm ${ }^{2}$ MSND is shown in Fig. (23). Notice in Fig. (23)that the pulse-height spectrum of the dual integrated detector shows a downward shift of the pulse-height signal compared to an individual 4- $\mathrm{cm}^{2}$ MSND. Some of this shift may be because of an overall increase in capacitance of the stacked MSNDs, thereby, reducing the pulse-height signal from the detector. For the stacked, large-area $4-\mathrm{cm}^{2}$, MSND design, with the LLD set to channel 18, just above the majority of the background signal, the intrinsic efficiency was measured to be $32.39 \pm 0.16 \%$. With the LLD raised to channel 28 , the intrinsic efficiency was measured to 
be $28.99 \pm 0.14 \%$.

The individual and stacked 4- $\mathrm{cm}^{2}$ large-area MSNDs were independently tested with a ${ }^{137} \mathrm{Cs}$ source with an activity of $71.69 \mathrm{mCi}$, to determine the gamma-ray detection efficiency, i.e., the $n / \gamma$ rejection ratio. The gamma-ray source was placed $50 \mathrm{~cm}$ away from the detector and a counting spectrum was taken. A background count was also taken to subtract any background counts and noise contributions. The gamma-ray rejection ratio was determined by dividing the integrated gamma-ray counts in the measured spectrum by the source gamma-ray fluence passing through the 4- $\mathrm{cm}^{2}$ MSND (adjusted for the solid angle subtended by the detector [70]). The gamma-ray pulse-height spectrum response is also shown in Fig. (23).

With the LLD set at channel 25, the intrinsic gamma-ray detection efficiency was determined to be approximately $1.85 \times 10^{-4} \%$, and with the LLD set to channel 32 the gamma-ray detection efficiency is approximately $3.95 \times 10^{-6} \%$. The neutron to gamma-ray rejection ratio $(n / \gamma)$ for $662-\mathrm{keV}$ photons is then easily calculated by dividing the MSND detection efficiency by the gamma-ray detection efficiency. The $n / \gamma$ ratio for the single MSND with an LLD set at channel 25 is $8.83 \times 10^{4}$, and with an LLD set at channel 32 the $\mathrm{n} / \gamma$ ratio is $4.04 \times 10^{6}$. Similar to the stacked $1-\mathrm{cm}^{2}$ MSND reported elsewhere [62], the deeper microstructured trench shows that the gammaray interaction pulse heights from the MSND are small, resulting in superior gamma-ray discrimination. Also, the individual 4- $\mathrm{cm}^{2}$ MSND has more bulk silicon with which gamma rays can interact than the $1-\mathrm{cm}^{2}$ detectors, yet the $n / \gamma$ ratio is better for the $4-\mathrm{cm}^{2}$ MSND because of a higher frequency of larger pulse heights from neutron interactions. Again, the MSND gammaray response measurement was performed in the KSU reactor bay, which has a higher background of scattered neutrons than the natural neutron background [71].

With the LLD set at channel 18, the gamma-ray detection efficiency for the stacked $4-\mathrm{cm}^{2}$ MSND was determined to be approximately $5.44 \times 10^{-3} \%$ and with the LLD set to channel 28, the gamma-ray detection efficiency is approximately $7.90 \times 10^{-6} \%$. The $n / \gamma$ response for $662 \mathrm{keV}$ photons is then easily calculated by dividing the stacked MSND detection efficiency by the gamma-ray detection efficiency. From Fig. (24), the $n / \gamma$ ratio for the stacked MSNDs with an LLD set at channel 18 is $5.95 \times 10^{3}$ and with an LLD set at channel 28 is $3.67 \times 10^{6}$. 


\section{Summary}

Based on the results and observations presented here, the following conclusions can be stated:

1. Calculations show that trench patterns provide stable efficiency over a wide range of LLD settings. Hole patterns have the best stability, but have lower efficiency. Hole patterns can be made to have higher efficiencies, but the remaining amount of semiconductor material is not enough to absorb much of the reaction product energies, thereby, shifting the pulse height spectrum to low energy channels. As a result, the higher efficiency hole pattern designs exhibit a large change in efficiency with changing LLD. Column patterns show the greatest change in efficiency with changing LLD settings. Overall, the trench pattern design offers the best performance when considering gammaray discrimination, intrinsic thermal-neutron detection efficiency, and efficiency stability over a wide range of LLD settings.

2. ${ }^{6} \mathrm{LiF}$ as a backfill material is more desirable as a neutron absorber than ${ }^{10} \mathrm{~B}$ because it permits higher LLD settings, thereby, allowing for larger $n / \gamma$ rejection ratios. Because of the larger ranges of the ${ }^{6} \mathrm{Li}$ reaction products, this material also allows larger device features than does ${ }^{10} \mathrm{~B}$, thereby, greatly facilitating device fabrication.

3. Etching is best performed with a highly anisotropic wet etch, such as performed with $\mathrm{KOH}$, mainly because the sidewall of the microstructures are left relatively undamaged. By contrast, plasma etching can be used for many different patterns, but causes sidewall damage that reduces detector performance. Because $\mathrm{KOH}$ wet etching is best performed with long straight features, the straight trench design is a superior choice for a micropattern.

4. Conformal diffusion of dopants into the MSND microstructures produces low leakage current and good detection performance.

5. Backfilling can be performed rapidly by centrifuging colloidal solutions of neutron reactive material into the microstructures. The method is performed at room temperature and does not damage the delicate microstructures.

6. Higher thermal-neutron detection efficiency with large-area MSNDs can be achieved by stacking the semiconductor detectors. The efficiencies for symmetric trench devices can be doubled by capturing streaming 
neutrons from the first detector. Microstructure patterns other than trench pattern variations do not conveniently stack to completely intersect neutron paths; hence trench patterns are best for stacked configurations.

7. With low LLD settings and minimal feature sizes, an individual MSND with asymmetric features (trenches wider than semiconductor fins) offers high efficiencies. Theoretically, asymmetric designs should have higher $\epsilon_{t n}$ [32]. However, with the LLD set at channels generally necessary to discriminate against background gamma rays, the stacked trench design with symmetric features outperforms the asymmetric designs (stacked or not).

At present, the highest efficiency recorded by an MSND is from a dual stack of $1-\mathrm{cm}^{2}$ devices, which demonstrated a best $\epsilon_{t n}$ of $42 \%$ [62]. The largest such devices, presented here, are $4-\mathrm{cm}^{2}$, dual stacked devices have shown a best $\epsilon_{t n}$ of $32.4 \%$. The next generation of devices will have straight trenches exceeding $450 \mu \mathrm{m}$ deep (as shown in Fig. (21), which should increase the $\epsilon_{t n}$ above $43 \%$. Overall, with the progress realized over the past decade, MSNDs are now at a stage of development ready for commercialization.

\section{Acknowledgements}

This work was supported in part by DTRA contract DTRA-01-03-C-0051, NSF grant no. 0412208, and DOE grant number DE-FG07-04ID14599.

\section{References}

[1] R.V. Babcock, R.E. Davis, S.L. Ruby, K.H. Sun, E.D. Wolley, Nucleonics, 17 (1959) pp. 116-122.

[2] Bibliographical Series No. 18, Neutron Detectors, International Atomic Energy Agency, Vienna, 1966 (references therein).

[3] H. Rauch, F. Grass, B. Feigl, Nucl. Instr. and Meth., 46 (1967) 153.

[4] A. Rose, Nucl. Instr. and Meth., 52 (1967) 166. 
[5] B. Feigl, H. Rauch, Nucl. Instr. and Meth., 61 (1968) 349.

[6] A.P. Jeavons, N.L. Ford, B. Lindberg, R. Sachot, Nucl. Instr. and Meth., 148 (1978) 29.

[7] P. Convert, J.B. Forsyth, Eds., Position-Sensitive Detection of Thermal Neutrons, (Academic Press, London, 1983).

[8] T. Aoyama, Y. Oka, K. Honda, C. Mori, Nucl. Instr. and Meth. A314 (1992) 635.

[9] A. Mireshghi, G. Cho, J.S. Drewery, T. Jing, S.N. Kaplan, V. Perezmendez, D. Wildermuth, IEEE Trans. Nucl. Sci., NS-39 (1992) 635.

[10] S. Pospisil, B. Sopko, E. Havrankova, Z. Janout, J. Konicek, I. Macha, J. Pavlu, Radiation Protection Dosimetry, 46 91993) 115.

[11] A. Mireshghi, G. Cho, J.S. Drewery, W.S. Hong, T. Jing, H. Lee, S.N. Kaplan, V. Perez-mendez, IEEE Trans. Nucl. Sci., NS-41 (1994) 915.

[12] R.L. Schulte, F. Swanson, M. Kesselman, Nucl. Instr. and Meth., A353 (1994) 123.

[13] D.S. McGregor, J.T. Lindsay, C.C. Brannon, and R.W. Olsen, Nucl. Instr.and Meth., A380 (1996) 271.

[14] D.S. McGregor, J.T. Lindsay, C.C. Brannon, and R.W. Olsen, IEEE Trans. Nucl. Sci., 43 (1996) 1358.

[15] J. Schelten, R. Reinartz, R. Engels, M. Balzhäuser, J. Lauter, W. Schäfer, K.D. Müller, Nucl. Instr. and Meth., A389 (1997) 447. 
[16] F. Foulon, P Bergonzo, A Brambilla, C. Jany, B. Guizard, R.D. Marshall, Proc. MRS, 487 (1998) 591.

[17] R.L. Schulte, M. Kesselman, Nucl. Instr. and Meth., A422 (1999) 852.

[18] D.S. McGregor, S.M. Vernon, H.K. Gersch, S.M. Markham, S.J. Wojtczuk and D.K. Wehe, IEEE Trans. Nucl. Sci., NS-47 (2000) 1364.

[19] D.S. McGregor, R.T. Klann, H.K. Gersch, and YH. Yang, Nucl. Instrum. and Meth., A466 (2001) pp. 126-141.

[20] D.S. McGregor, H.K. Gersch, J.D. Sanders, R.T. Klann, and J.T. Lindsay, J. Korean Asso. for Rad. Prot., 26 (2001) 167.

[21] H.K. Gersch, D.S. McGregor, and P.A. Simpson, Nucl. Instrum. and Meth., A489 (2002) 85.

[22] D.S. McGregor, R. T. Klann, J. D. Sanders, J.T. Lindsay, K.J. Linden, H.K. Gersch, P.M. De Lurgio, C.L. Fink, and Elsa Ariesanti, Proc. of SPIE, Vol. 4784 (2002) 164.

[23] D.S. McGregor, M.D. Hammig, Y-H Yang, H.K. Gersch, R.T. Klann, Nucl. Instr. and Meth., A500 (2003) 272.

[24] D.I. Garber, R.R. Kinsey, BNL 325: Neutron Cross Sections, 3rd ed., Vol. 2, Curves, Brookhaven National Laboratory, Upton (1976).

[25] V. McLane, C.L. Dunford, P.F. Rose, in: Neutron Cross Sections, Vol. 2, Academic Press, San Diego, 1988. 
[26] D.S. McGregor, R.T. Klann, H.K. Gersch, E. Ariesanti, J.D. Sanders, and B. Van Der Elzen, Conf. Rec. IEEE Nucl. Sci. Symp., San Diego, California, Nov. 4-9, 2001.

[27] R.A. Muminov, L.D. Tsvang, Sov. At. Energy, 62 (1987) 255.

[28] J. Schelten, M. Balzhäuser, F. Höngesberg, R. Engels, R. Reinartz, Physica B, 234-236 (1997) 1084.

[29] J. Schelten, R.Reinartz, Neutron Detector, US Patent 58804762, allowed March 9, 1999.

[30] J.K. Shultis and D.S. McGregor, Conf. Rec. IEEE Nucl. Sci. Symp., Rome, Italy, Oct. 18-22, 2004.

[31] J.K. Shultis and D.S. McGregor, IEEE Trans. Nucl. Sci., NS-53 (2006) 1659.

[32] J.K. Shultis, D.S. McGregor, Nucl. Instr. and Meth., A606 (2009) 608.

[33] D.S. McGregor, R.T. Klann, H.K. Gersch, E. Ariesanti, J.D. Sanders, and B. Van Der Elzen, IEEE Trans. Nucl. Sci., 49 (2002) 1999.

[34] D.S. McGregor and R.T. Klann, US Patent 6545281; allowed April 8, 2003.

[35] R.J. Nikolic, C.L. Cheung, C.E. Reinhardt, T.F. Wang, Proc. SPIE, Photonics West, Boston, MA (2005). 
[36] W.J. McNeil, S. Bellinger, T. Unruh, E. Patterson, A. Egley, D. Bruno, M. Elazegui, A. Streit, D.S. McGregor, Conf. Rec. IEEE Nucl. Sci. Symp., San Diego, CA, Oct. 29-Nov. 3, 2006.

[37] R.J. Nikolic, C.L. Cheung, C.E. Reinhardt, T.F. Wang, "Future of Semiconductor Based Thermal Neutron Detectors," Proc. NSTI Nanotech 2006, Boston, MA (2006).

[38] S.L. Bellinger, W.J. McNeil, T.C. Unruh, D.S. McGregor, Conf. Rec. IEEE Nucl. Sci. Symp., Waikiki, Hawaii, Oct. 28-Nov. 3, 2007.

[39] C.J. Solomon, J.K. Shultis, W.J. McNeil, T.C. Unruh, B.B. Rice, and D.S. McGregor, Nucl. Instrum. and Meth., A580 (2007) 326.

[40] D.S. McGregor, S.L. Bellinger, D. Bruno, S. Cowley, M. Elazegui, W.J. McNeil, E. Patterson, B.B. Rice, C.J. Solomon, J.K. Shultis, and T. Unruh, Conf Rec. IEEE Conf. on Tech. for Homeland Security, Woburn, MA, May 16-17, 2007.

[41] D.S. McGregor, S.L. Bellinger, D. Bruno, W.J. McNeil, E. Patterson, J.K. Shultis, C.J. Solomon, T. Unruh, Proc. SPIE, 6706 (2007) 0 N1.

[42] D.S. McGregor, R.T. Klann, patent US-7,164,138; allowed January 16, 2007.

[43] W.J. McNeil, S.L. Bellinger, B.J. Blalock, C.L. Britton Jr., J.L. Britton, S.C. Bunch, W.L. Dunn, C.M. Henderson, T.J. Sobering, R.D. Taylor, T.C. Unruh, D. S. McGregor, Conf. Rec. IEEE Nucl. Sci. Symp., Waikiki, Hawaii, Oct. 28-Nov. 3, 2007.

[44] C.J. Solomon, J.K. Shultis, W.J. McNeil, T.C. Unruh, B.B. Rice, and D.S. McGregor, Nuclear Instruments and Methods, A580 (2007) 326. 
[45] Q. Jahan, E. Patterson, B. Rice, W.L. Dunn, D.S. McGregor, Nucl. Instr. and Meth., B263 (2007) 183.

[46] J. Uher, F. Frojdh, J. Jakubek, C. Kenney, Z. Kohout, V. Linhart, S. Parker, S. Peterson, S. Poposil, G. Thungstron, Nucl. Instr. and Meth., A576 (2007) 32.

[47] D.S. McGregor, J.K. Shultis, Proc SPIE, 7079 (2008) 06-1.

[48] N. LiCausi, J. Dingley, Y. Danon, J-Q Lu, I.B. Bhat, Proc SPIE, 7079 (2008) 08-1.

[49] R.J. Nikolic, A.M. Conway, C.E. Reinhardt, R.T. Graff, T.F. Wang, N. Deo, C.L. Cheung, Appl. Phys. Lett., 93 (2008) 133502.

[50] D.S. McGregor, S.L. Bellinger, W.J. McNeil, T.C. Unruh, Conf. Rec. IEEE Nucl. Sci. Symp., Dresden, Germany, Oct. 19-Oct. 25, 2008.

[51] S.L. Bellinger, W.J. McNeil and D.S. McGregor, Proc. MRS, vol. 1164 (2009) L06.

[52] S.L. Bellinger, W.J. McNeil, T.C. Unruh, D.S. McGregor, IEEE Trans. Nucl. Sci., 56 (2009) 742.

[53] D.S. McGregor, W.J. McNeil, S.L. Bellinger, T.C. Unruh, J.K. Shultis, Nucl. Instrum. and Meth. A608 (2009) 125.

[54] D.S. McGregor, S.L. Bellinger, D. Bruno, W.L. Dunn, W.J. McNeil, E. Patterson, B.B. Rice, J.K. Shultis, T. Unruh, Rad. Phys. and Chem., 78 (2009) 874. 
[55] C.J. Solomon, J.K. Shultis, D.S. McGregor, Nucl. Instr. and Meth., A618 (2010) 260.

[56] C.M. Henderson, Q.M. Jahan, W.L. Dunn, J.K. Shultis and D.S. McGregor, Rad. Phys. and Chem., 79 (2010) 144.

[57] D.S. McGregor, S.L. Bellinger, W.J. McNeil, E.L. Patterson, B.B. Rice, J.K. Shultis, C.J. Solomon, US Patent 7855372; allowed December 21, 2010 .

[58] S.L. Bellinger, R.G. Fronk, W.J. McNeil, J.K. Shultis, T.J. Sobering, D.S. McGregor, Proc. SPIE, 7805 (2010) 7805-0N.

[59] S.L. Bellinger, R.G. Fronk, W.J. McNeil, T.J. Sobering, and D.S. McGregor, Nucl. Instrum. and Meth., A 652 (2011) 387.

[60] S.L. Bellinger, Advanced Microstructured Semiconductor Neutron Detectors: Design, Fabrication, and Performance, Ph.D. Dissertation, Kansas State University, 2011.

[61] Y. Danon, J. Clinton, K.C. Huang, N. LiCausi, R. Dahal, J.J.Q. Lu and I. Bhat, 2nd Int. Workshop on Fast Neutron Detectors and Applications, Nov. 6-11, 2011, Ein Gedi, Israel.

[62] S.L. Bellinger, R.G. Fronk, W.J. McNeil, T.J. Sobering, D.S. McGregor, IEEE Trans. Nucl. Sci., 59 (2012) 167.

[63] S.L. Bellinger, R.G. Fronk, T.J. Sobering, D.S. McGregor, Proc. SPIE, 8373 (2012) OI-1.

[64] R. Dahal,K. C. Huang, J. Clinton,2 N. LiCausi, J.-Q. Lu, Y. Danon, and I. Bhat, Appl. Phys. Lett., 100 (2012) 243507. 
[65] B.B. Rice, Inductively Coupled Plasma Etching of Silicon and Gallium Arsenide, M.S. Thesis, Kansas State University, 2006.

[66] K.E. Bean, IEEE Trans. Elec. Devices, ED-25 (1978) 1185.

[67] H. Lim, Y. Kim, Jpn. J. Appl. Phys., 37 (1998) 7086.

[68] D.L. Kendall, G.R. de Guel, Micromachining and Micropackaging of Transducers, (Elsevier: Amsterdam) 1985.

[69] S.L. Bellinger, R.G. Fronk, D.S. McGregor, US Patent Pending (2012).

[70] D.S. McGregor, J.K. Shultis, Nucl. Instrum. and Meth., A608 (2011) 167.

[71] M.S. Gordon, P. Goldhagen, K.P. Rodbell, T.H. Zabel, H.H.K. Tang, J.M. Clem, P. Bailey, IEEE Trans. Nucl. Sci., 51 (2004) 3427.

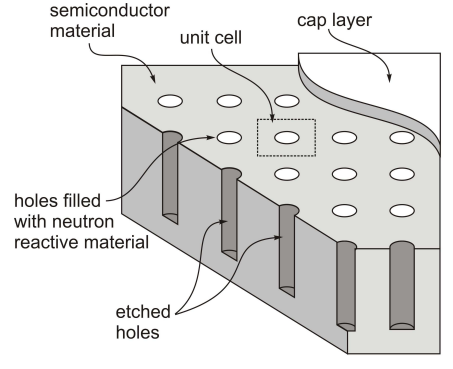

(a)

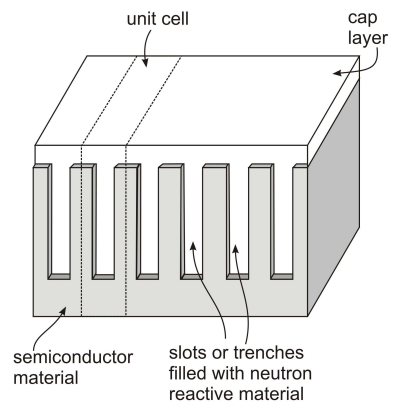

(b)

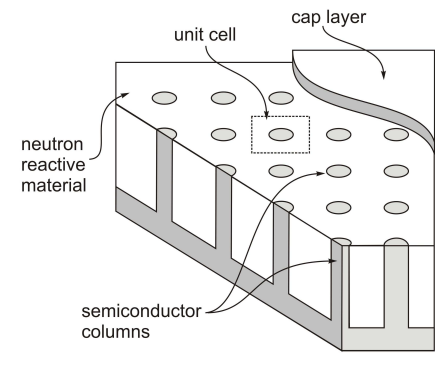

(c)

Figure 1: Three basic microstructures use a matrix of (a) holes, (b) trenches (or semiconductor fins), or (c) semiconductor columns. 


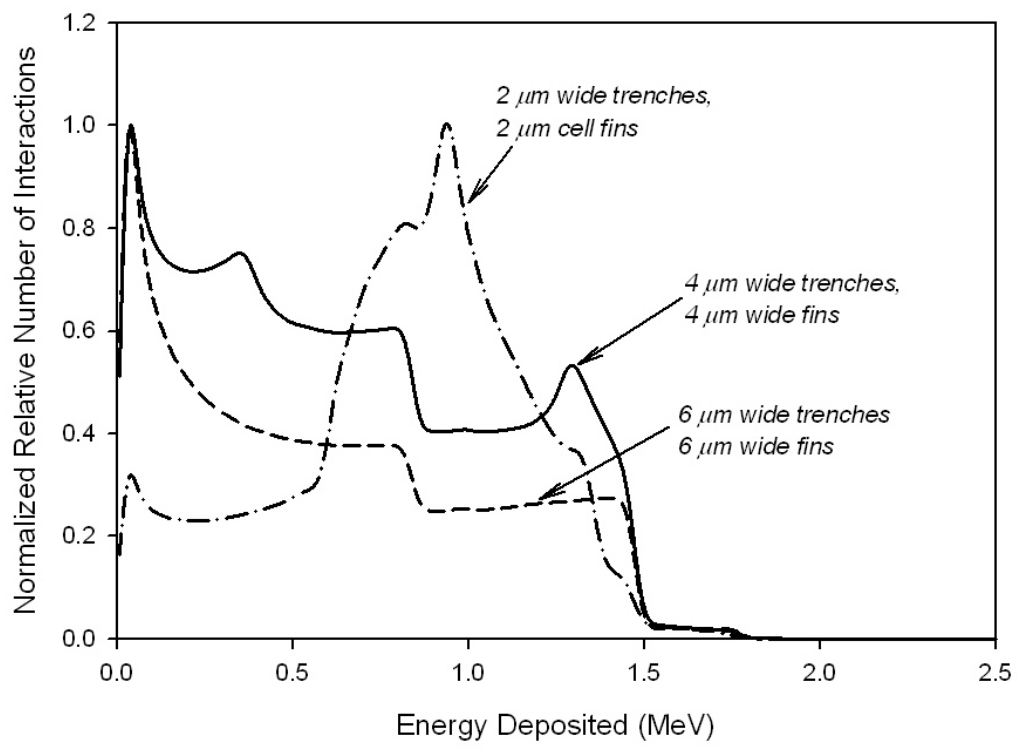

Figure 2: Modeled spectra for $40 \mu \mathrm{m}$ deep ${ }^{10} \mathrm{~B}$ backfilled trench pattern devices for the cases in which the trench widths are $2 \mu \mathrm{m}, 4 \mu \mathrm{m}$, or $6 \mu \mathrm{m}$ wide, each with a $50 \%$ cell fraction. The spectra are normalized to the highest point of each spectrum and show the relative normalized number of events per unit energy deposited. 


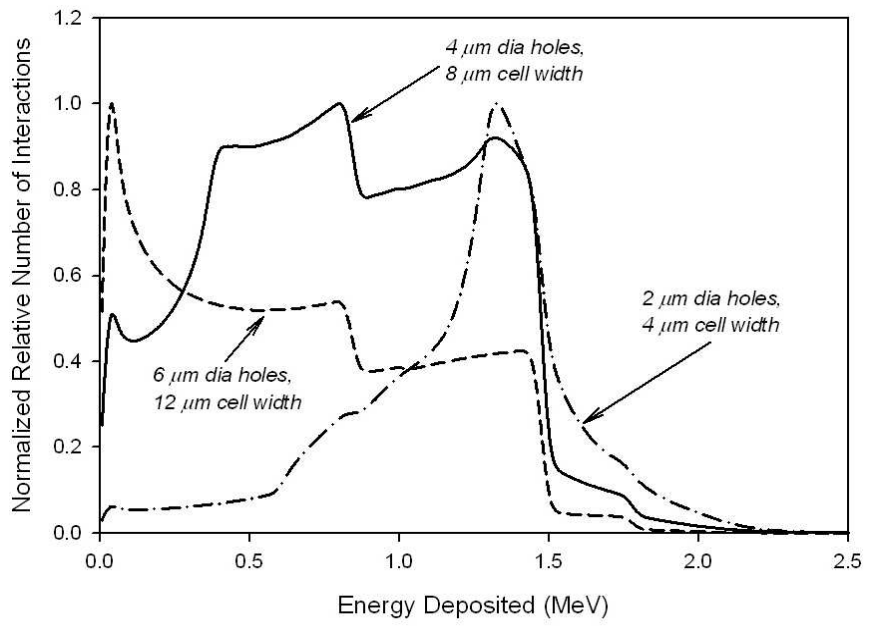

Figure 3: Modeled spectra for $40 \mu \mathrm{m}$ deep ${ }^{10} \mathrm{~B}$ backfilled hole pattern devices for the cases in which the hole diameters are $2 \mu \mathrm{m}, 4 \mu \mathrm{m}$, or $6 \mu \mathrm{m}$ wide, each with a $50 \%$ cell fraction. The spectra are normalized to the highest point of each spectrum and show the relative normalized number of events per unit energy deposited.

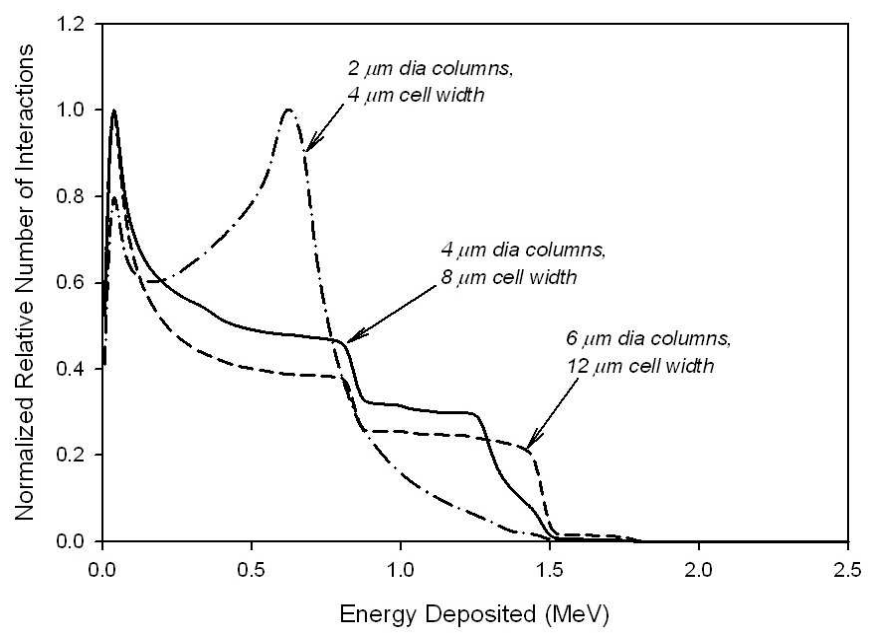

Figure 4: Modeled spectra for $40 \mu \mathrm{m}$ deep ${ }^{10} \mathrm{~B}$ backfilled column pattern devices for the cases in which the column diameters are $2 \mu \mathrm{m}, 4 \mu \mathrm{m}$, or $6 \mu \mathrm{m}$ wide, each with a $50 \%$ cell fraction. The spectra are normalized to the highest point of each spectrum and show the relative normalized number of events per unit energy deposited. 


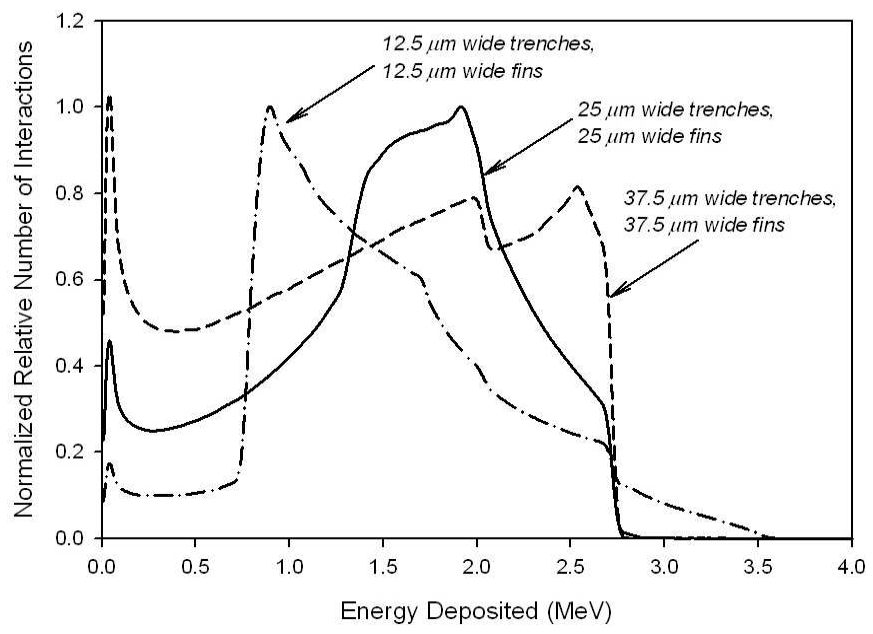

Figure 5: Modeled spectra for $350 \mu \mathrm{m}$ deep ${ }^{6} \mathrm{LiF}$ backfilled trench pattern devices for the cases in which the trench widths are $12.5 \mu \mathrm{m}, 25 \mu \mathrm{m}$, or $37.5 \mu \mathrm{m}$ wide, each with a $50 \%$ cell fraction. The spectra are normalized to the highest point of each spectrum and show the relative normalized number of events per unit energy deposited.

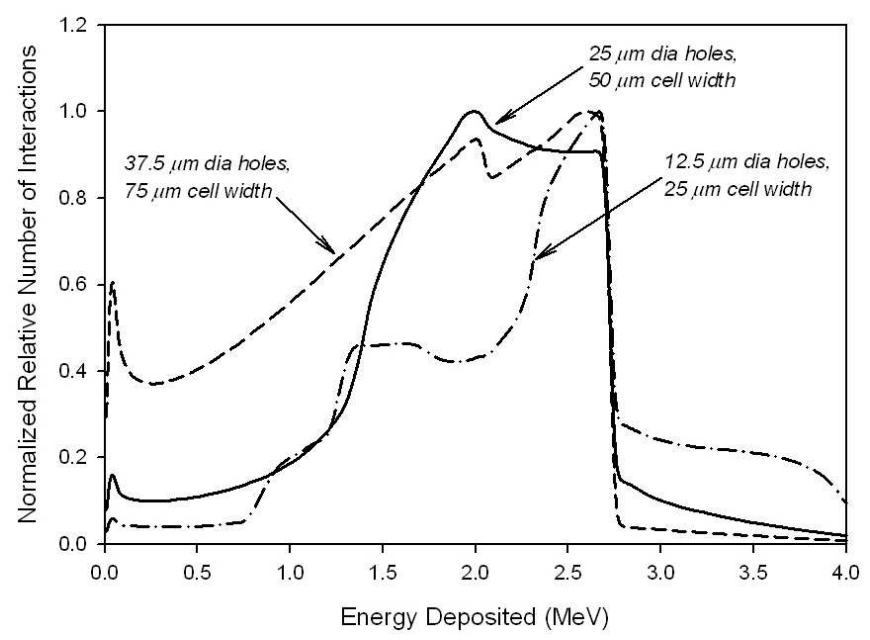

Figure 6: Modeled spectra for $350 \mu \mathrm{m}$ deep ${ }^{6} \mathrm{LiF}$ backfilled hole pattern devices for the cases in which the hole diameters are $12.5 \mu \mathrm{m}, 25 \mu \mathrm{m}$, or $37.5 \mu \mathrm{m}$ wide, each with a $50 \%$ cell fraction. The spectra are normalized to the highest point of each spectrum and show the relative normalized number of events per unit energy deposited. 


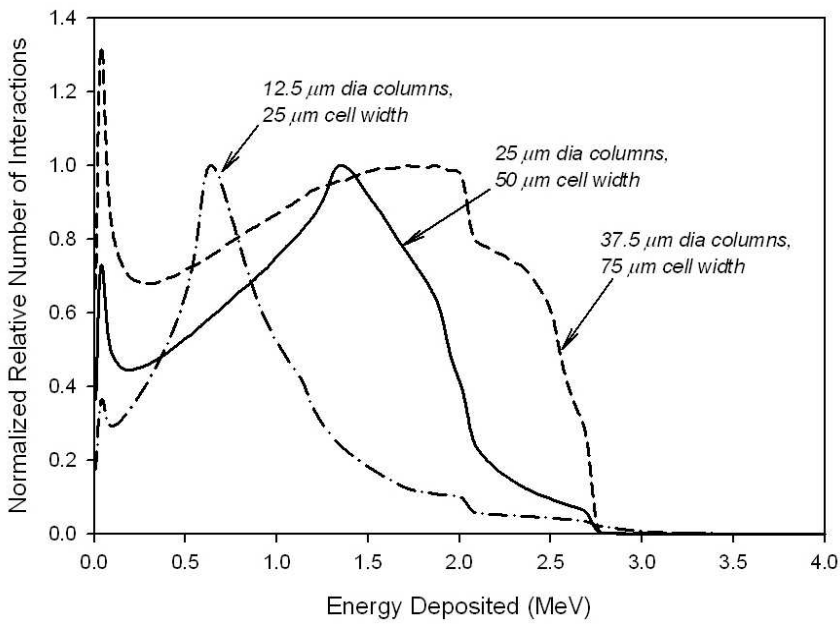

Figure 7: Modeled spectra for $350 \mu \mathrm{m}$ deep ${ }^{6} \mathrm{LiF}$ backfilled column pattern devices for the cases in which the column diameters are $12.5 \mu \mathrm{m}, 25 \mu \mathrm{m}$, or $37.5 \mu \mathrm{m}$ wide, each with a $50 \%$ cell fraction. The spectra are normalized to the highest point of each spectrum and show the relative normalized number of events per unit energy deposited.

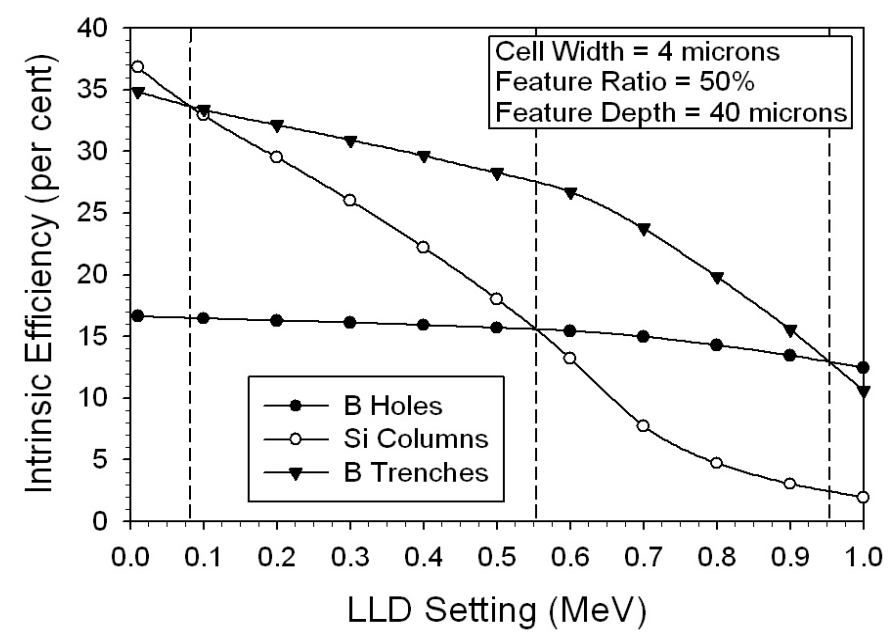

Figure 8: Comparison of $\epsilon_{t n}$ as a function of feature size, as measured by its cell fraction, for hole, trench and column designs with unit cell dimensions of $4 \mu \mathrm{m}$ and feature depths of $40 \mu \mathrm{m} .{ }^{10} \mathrm{~B}$ is the backfill material and the LLD was set for $300 \mathrm{keV}$. 


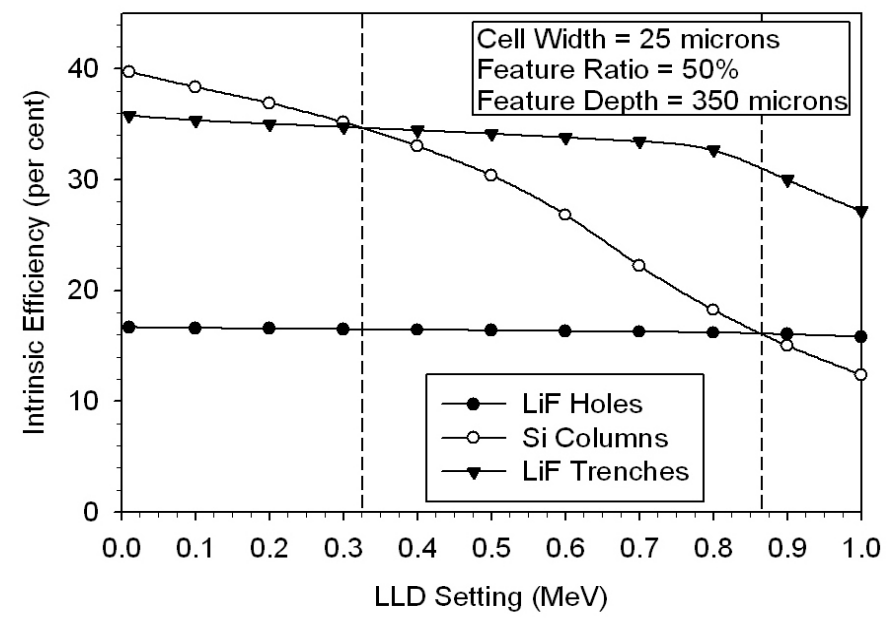

Figure 9: Comparison of $\epsilon_{t n}$ as a function of feature size, as measured by its cell fraction, for hole, trench and column designs with unit cell dimensions of $40 \mu \mathrm{m}$ and feature depths of $350 \mu \mathrm{m} .{ }^{6} \mathrm{LiF}$ is the backfill material and the LLD was set for $300 \mathrm{keV}$. 


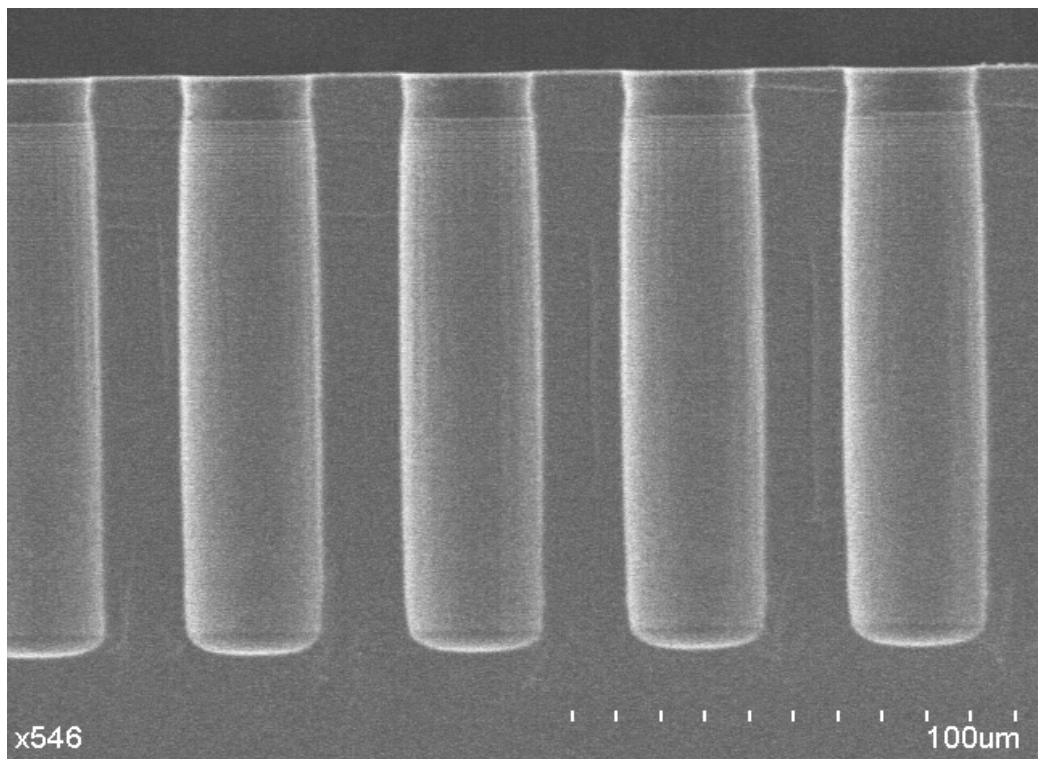

Figure 10: An example of the bottling effect from ICP-RIE. Botting causes the microstructure to be small at the top and larger at the bottom, which can make backfilling difficult.

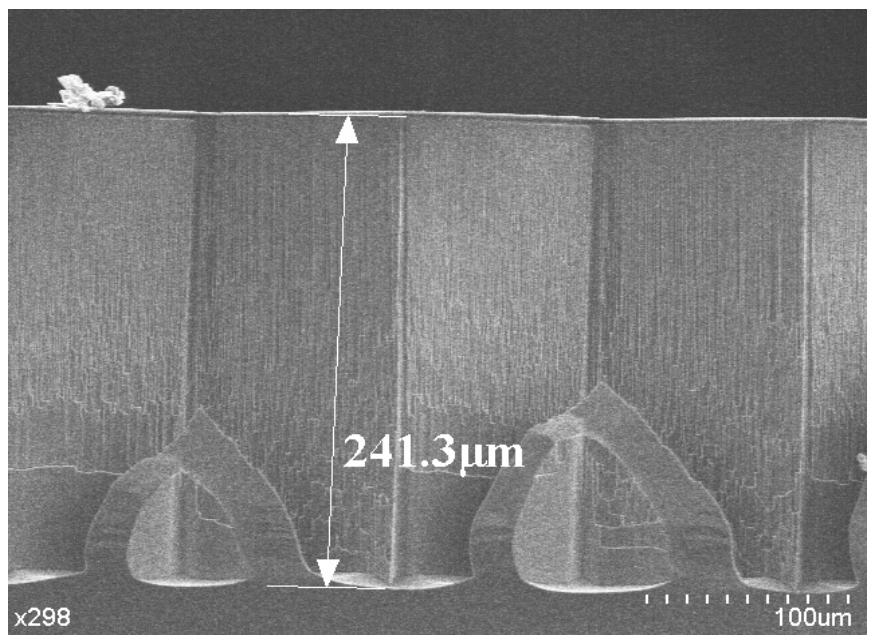

Figure 11: An example of the scalloping effect from ICP-RIE. Scalloping damages the sidewalls which can lead to excessive leakage current. 


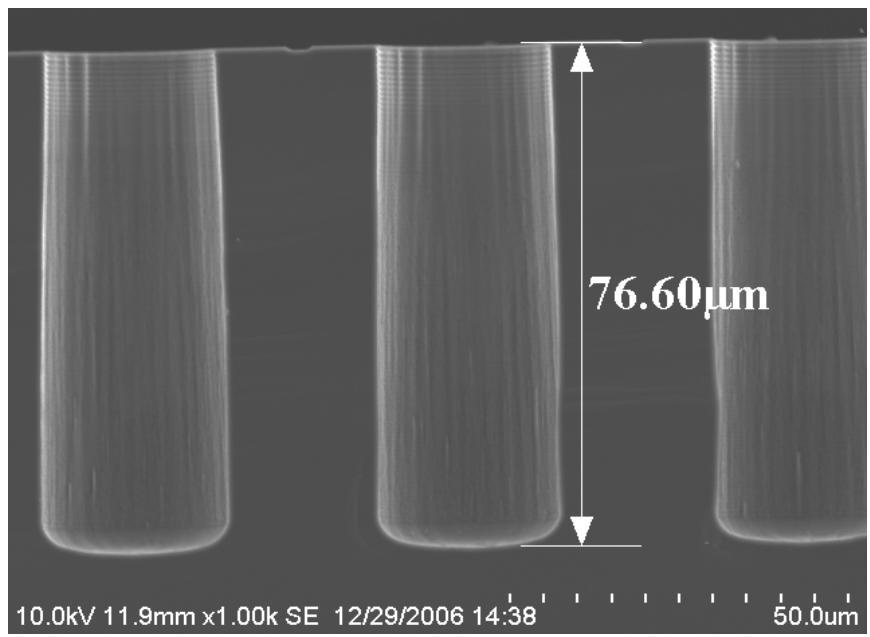

Figure 12: An example of the striations from ICP-RIE. Striations damage the sidewalls and can cause the appearance of pinnacles; both can lead to excessive leakage current.

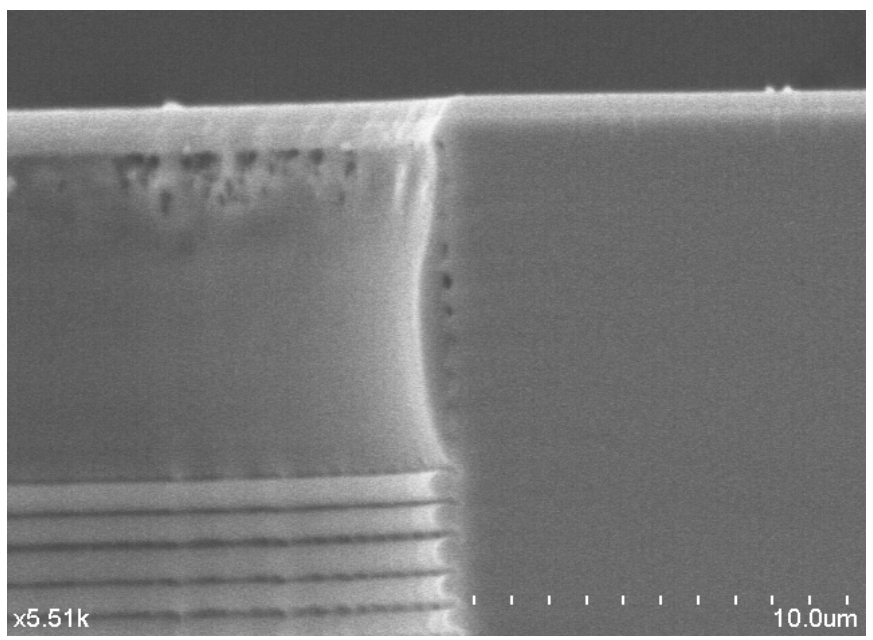

Figure 13: Ribbing is caused from the cyclic nature of deep etching with ICP-RIE. The damaged sidewalls can lead to excessive leakage current. 


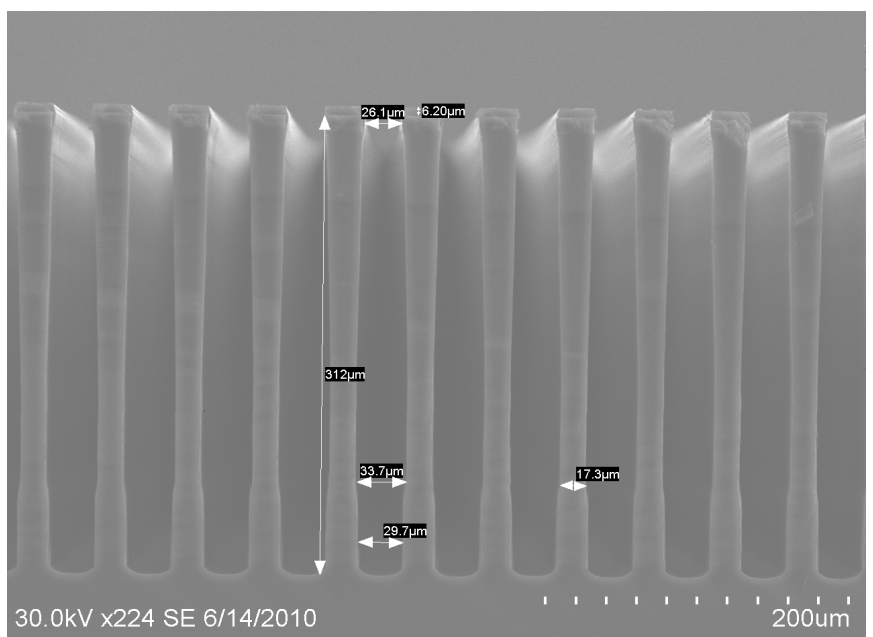

Figure 14: ICP-RIE deep etch showing $312 \mu \mathrm{m}$ deep smooth sidewalls, a preferred result for MSNDs. Bottling is also apparent.

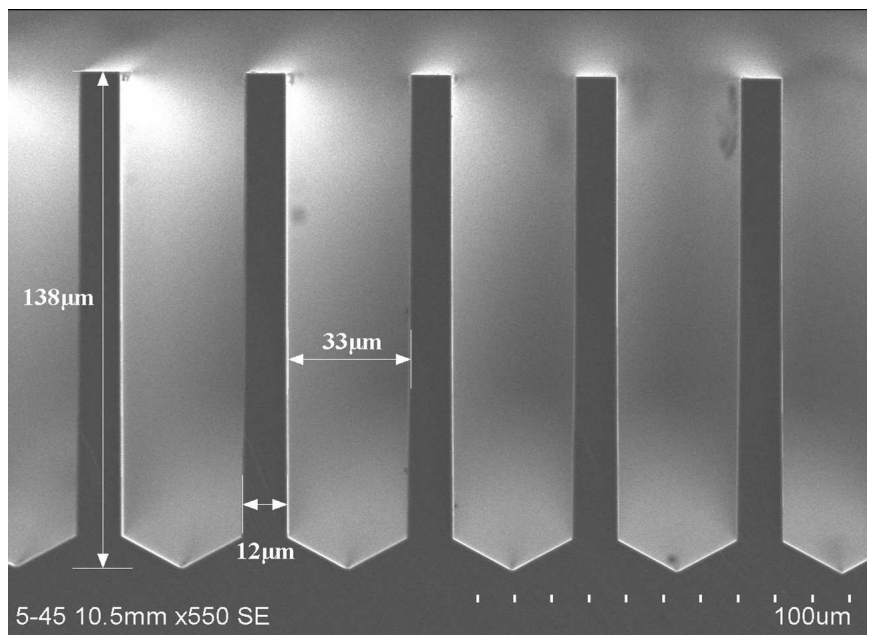

Figure 15: KOH etch of Si with $138 \mu \mathrm{m}$ deep straight trenches, showing smooth sidewalls that are preferred for MSNDs. 


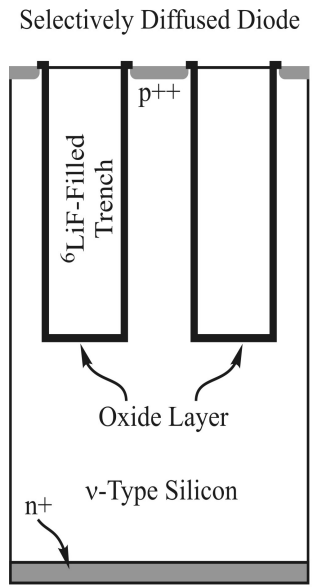

(a)
Conformally Diffused Diode

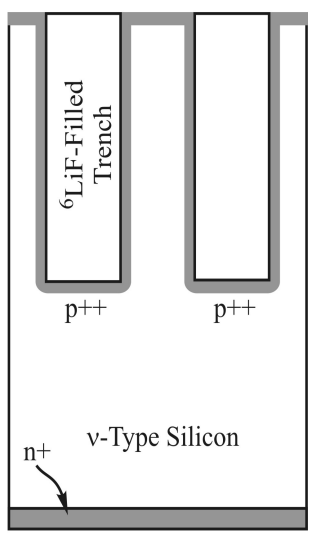

(b)

Figure 16: Cross-sectional view of (a) the selective-diffused perforated diode structure with side-wall passivation and (b) the conformal-diffused perforated diode structure. 


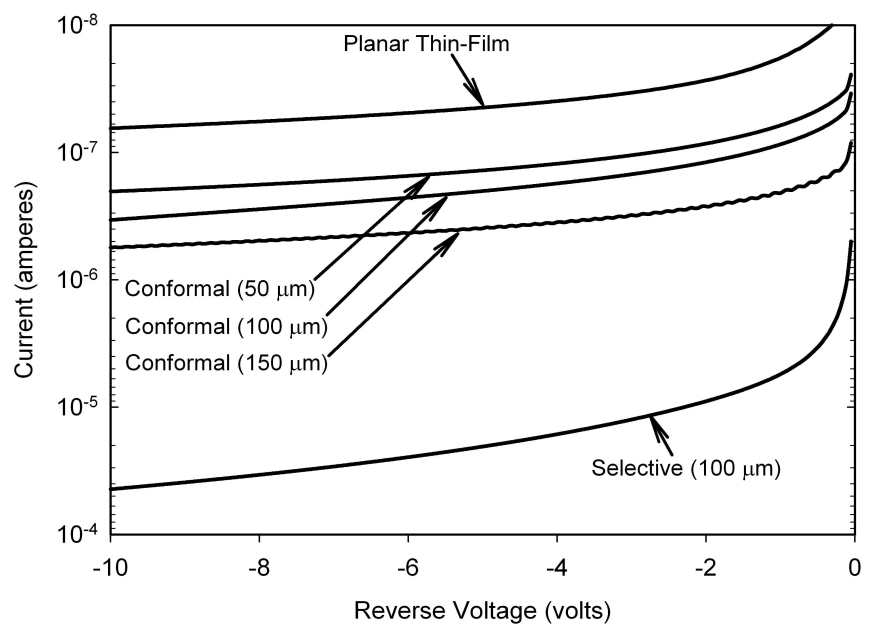

Figure 17: Leakage current from a planar thin-film-coated detector, a selective-diffused diode with $100 \mu \mathrm{m}$ deep trenches, and three conformal-diffused diodes with 50, 100 and $150 \mu \mathrm{m}$ deep trenches. All devices were $6 \mathrm{~mm}$ in diameter.

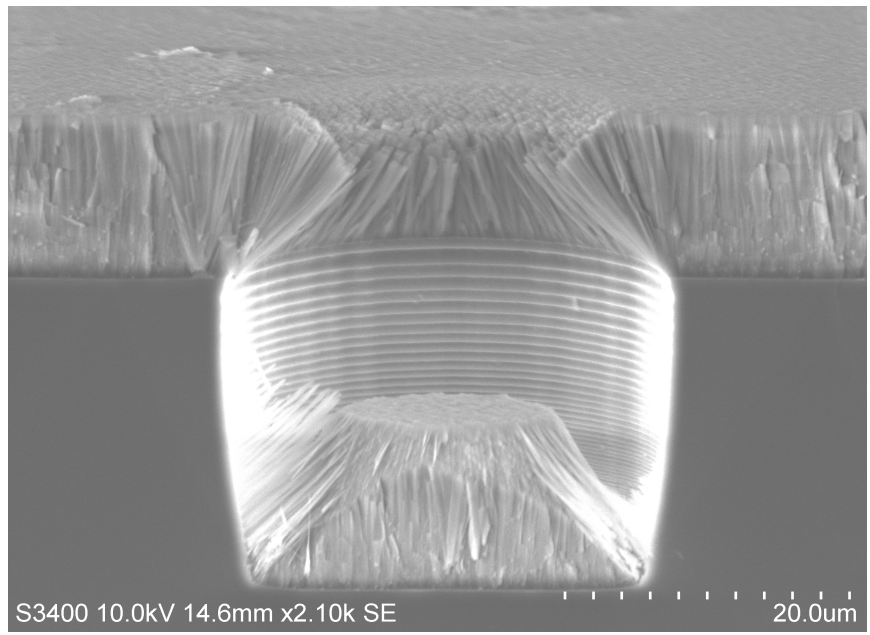

Figure 18: Physical vapor deposition (evaporation) of LiF within a hole feature, showing the pinch-off problem. 


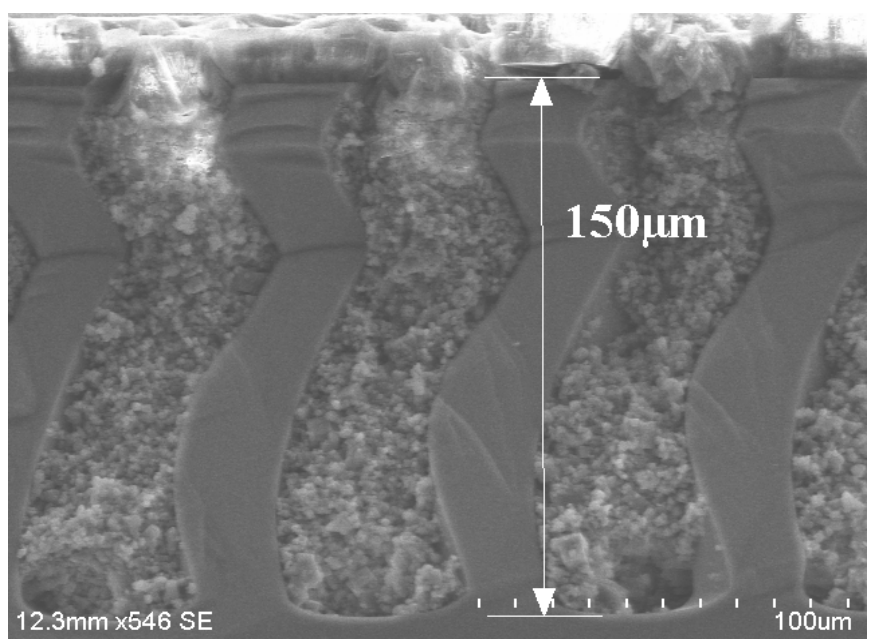

Figure 19: Hand packed LiF in a sinusoidal trench.

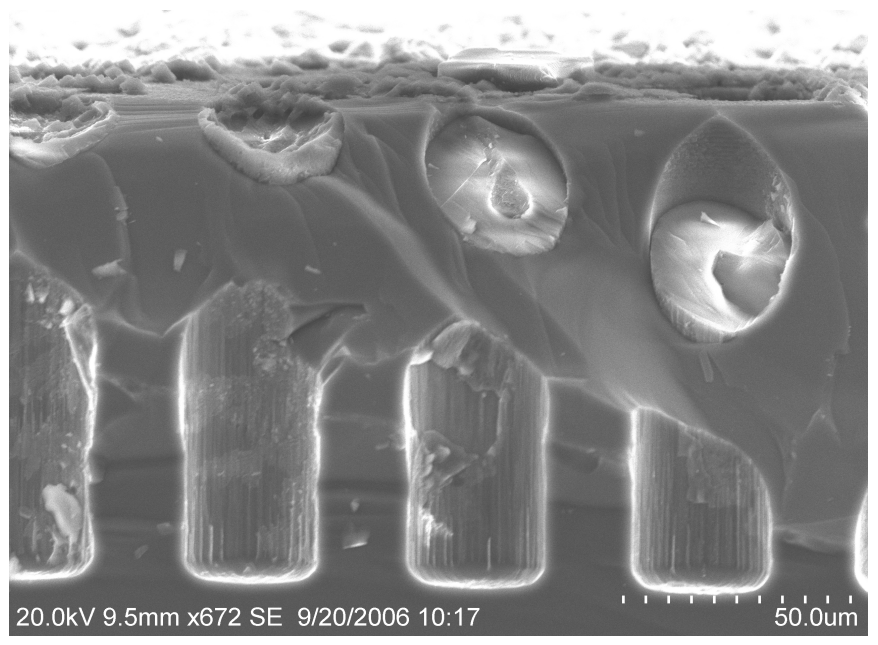

Figure 20: Melt conditioning of LiF in a hole pattern device. 


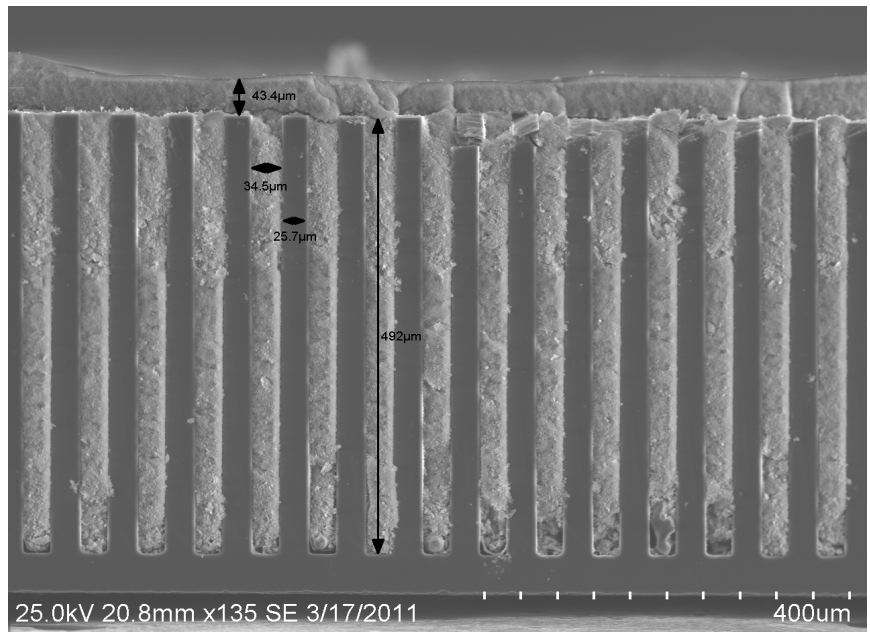

Figure 21: Centrifugal filling of colloidal $\mathrm{LiF}$ in $492 \mu \mathrm{m}$ deep straight trenches. The method is fast, inexpensive, completely fills the microstructures, and does not damage the device.

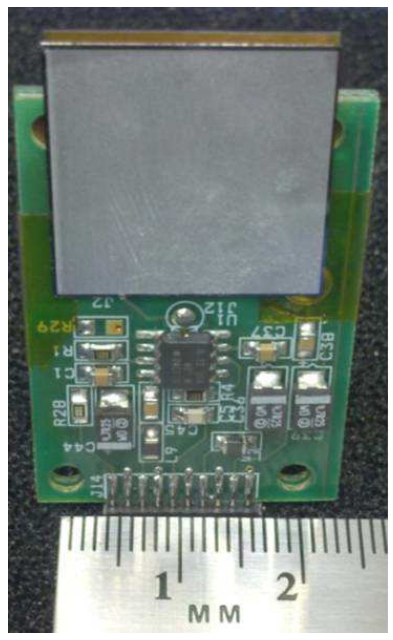

Figure 22: A 4- $\mathrm{cm}^{2}$, trench pattern, dual-integrated, stacked, microstructured semiconductor neutron detector with preamplifying circuitry. These dual stacked devcies are the largest and highest efficiency MSNDs currently available. 


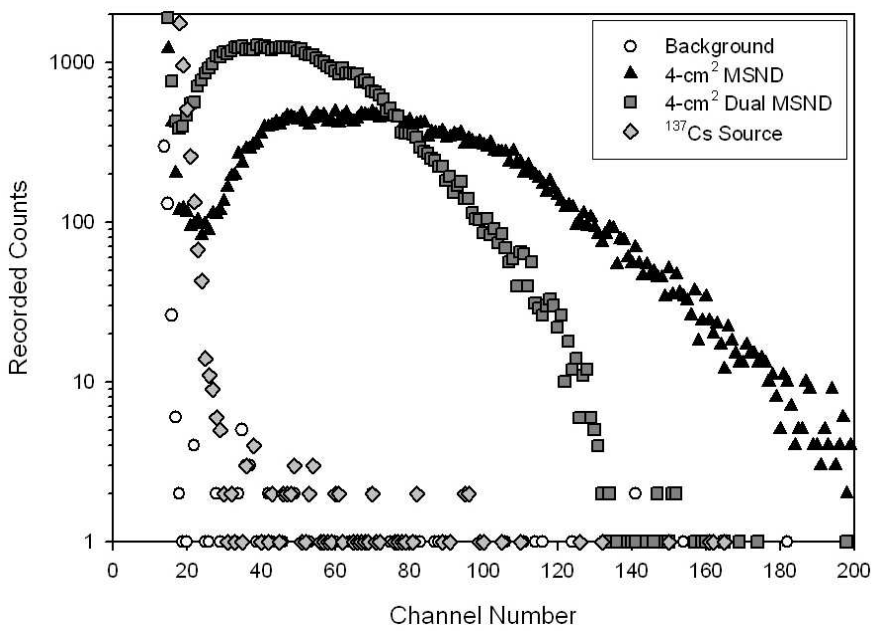

Figure 23: Comparison of pulse-height spectra from an individual and a stacked 4- $\mathrm{cm}^{2}$ MSND, each with $200 \mu \mathrm{m}$ deep, straight trench microstructures backfilled with ${ }^{6} \mathrm{LiF}$. Note the low number of counts in the low energy region. The background was measured with a $\mathrm{Cd}$ shield to block thermal neutrons. The response to a $71.69-\mathrm{mCi}{ }^{137} \mathrm{Cs}$ gamma-ray source is also shown. The gamma ray rejection ratio was measured to be $3.67 \times 10^{6}$ with the LLD set at channel 28.

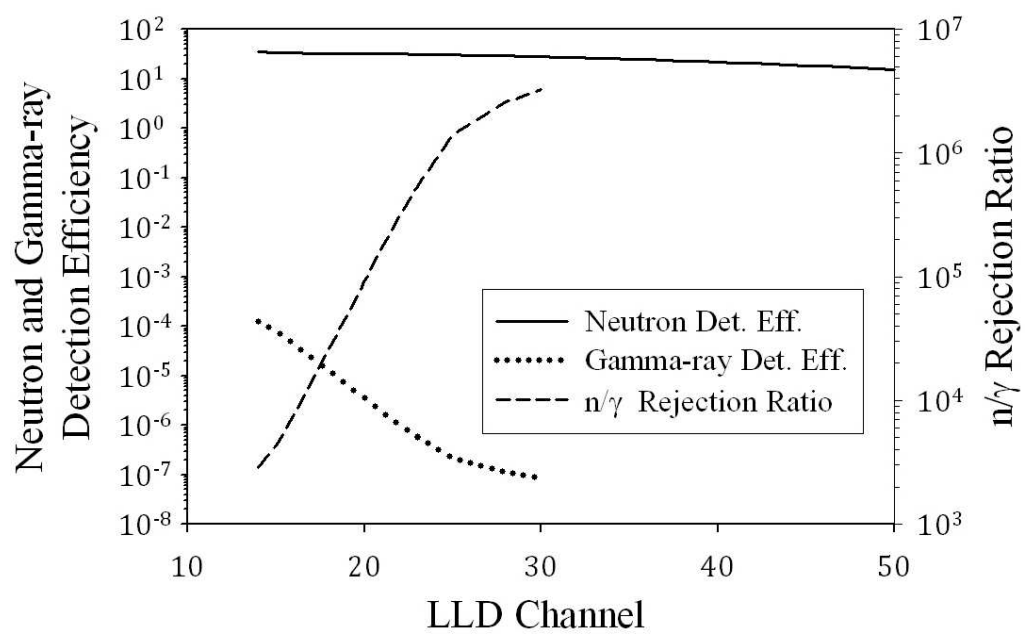

Figure 24: Comparison of the measured neutron detection efficiency, the measure ${ }^{137} \mathrm{Cs}$ gamma-ray detection efficiency, and the $\mathrm{n} / \gamma$ rejection ratio of the stacked $4-\mathrm{cm}^{2}$ MSND. 